\title{
Minimax Control of Distributed Discrete Time Systems through Spectral Factorization
}

\author{
Jarmo Malinen \\ Institute of Mathematics \\ Helsinki University of Technology \\ P. O. Box 1100 \\ FIN-02015 HUT, Finland
}




\begin{abstract}
In this paper we introduce a Riccati equation theory for (a class of) well posed (I/O-stable) discrete time linear systems $\Phi$ as presented in [9].

We tie together three different notions: The first notion is the general question under which conditions it is possible to solve a minimax control problem associated to $\Phi$ by static state feedback. The second notion concerns the existence of a certain spectral factorization of the I/O-map of $\Phi$. The third notion is about a particular (stabilizing) solution of a Riccati equation system associated with $\Phi$.

We show that these three notions are in fact equivalent under fairly mild stability assumptions of $\Phi$, namely input-output stability. Furthermore, this equivalence does not require any finite dimensional structure in any of the operators of the system.
\end{abstract}

AMS Subject Classification 93B52, 49J35, 93B36.

Keywords Discrete time, feedback control, infinite dimensional, input-output stable, minimax, Riccati, (J,S)-inner-outer factorization. 


\section{Introduction}

This paper, together with [9], presents a Riccati equation theory for a class of discrete time linear systems (DLS's) $\Phi$ with $H^{\infty}$ transfer functions. Complete and detailed proofs of the important results are given.

We study certain feedback properties of such linear systems. We show that the following three notions are equivalent:

(i) The (critical) control input giving the minimax output for $\Phi$ can be realized by a state feedback with a bounded feedback operator $K^{\text {crit }}$,

(ii) The transfer function $\mathcal{D}(z)$ of $\Phi$ has a $(J, S)$-inner-outer factorization as defined in Definition 18,

(iii) There is a sesquilinear form $P($, ) satisfying the Riccati equation of Definition 33 and certain additional conditions as listed in (iii) of Theorem 40.

For the precise statement of the results, see Theorem 40. For a brief presentation, see [8] which is a shorter version of this paper.

The results of this paper do not require any finite dimensional structure in any of the spaces. The cost functional in the output space $\Phi$ can be non-standard - i.e. also negative cost is allowed (see Definition 1). We use fairly weak stability conditions: The transfer function $\mathcal{D}(z)$ of the open loop system is in $H^{\infty}$, and the critical (one step) feedback operator $K^{\text {crit }}$ (see Definition 7 ) is assumed to be bounded. The latter condition is trivially satisfied if the system is output stable, or if the input space $U$ is finite dimensional. The controllability and observability maps of $\Phi$ may be unbounded. For this reason, the Riccati equation in Definition 33 is not stated in terms of a bounded self-adjoint Riccati operator but in terms of densely defined sesquilinear forms in the space $H \times H$, where $H$ is the state space of the system.

Let us give a short review of related material with emphasis on discrete time systems. Early papers about spectral factorization techniques, feedback control and stabilizing solutions of Riccati equations are [5], [12] and [16] for discrete time, and [11] and [13] for continuous time.

Equivalence results of type (ii) $\Leftrightarrow$ (iii) are given in [6] for finite dimensional systems both in continuous and discrete time. Also the notion of the extended Hamiltonian pencil (EHP) is introduced, and the equivalence of the feedback problem to an invariant subspace structure of EHP is studied (see also [15]). Discrete time EHP in the infinite dimensional setting is studied in [14] and existence results for (power) a stabilizing solution of the Riccati equation are given.

The monograph [4] contains a Riccati equation theory for exponentially (power) stable time-varying discrete time systems. The power stabilizing solution to the Riccati equation, 
minimax cost problems and factorizations of the transfer function are studied in terms of Kalman-Szegö-Popov-Yakubovich systems. The main emphasis is on the Riccati equation arising from the disturbance attenuation problem. A comprehensive reference for the classical finite dimensional case with positive cost functional [7] . Both continuous and discrete time systems are extensively treated from the Riccati equation point of view. The finite dimensional discrete time $H^{\infty}$-control problem is studied in [25] in terms of the Riccati equation and the (power) stabilizing solution. Some infinite dimensional discrete time Riccati equation theory is presented in [2].

The litterature for the continuous time case is considerably richer. Recent continuous time papers, somewhat parallelling our work, are [1], [6], [19], [21], [22], [24], [20], [29], [10]. The papers [1], [6] contain also short reviews of the history and development of the theories connecting the spectral factorization and feedback control; the latter for the discrete time systems, too.

The general organization of this paper is as follows. A crash course in discrete time linear systems (DLS's) is given in section 2. In section 3 we define and prove basic facts about a minimax control problem of I/O-stable DLS's. Section 4 is devoted to the study of $(J, S)$ inner-outer factorizations of the I/O-map $\mathcal{D}$ and $S$-spectral factorizations of the Popov operator. In section 5 we show that the minimax problem can be solved in feedback form if and only if $\mathcal{D}$ has a $(J, S)$-inner-outer factorization (see Theorem 27 ). Under the same conditions it is true that the sesquilinear form describing the critical cost satisfies a Riccati equation of Definition 33; this is shown in section 6. The converse result is given in section 7: the existence of a particular solution of the same Riccati equation implies the equivalent conditions of Theorem 27. Finally, in section 8 , the three equivalent conditions are collected in our main Theorem 40 and some existence results for the $(J, S)$-inner-outer factorizations are discussed.

\section{A short review of DLS's}

We review the structure and notations of [9] that will be used throughout this paper.

The following notations are used throughout the paper: $\mathbf{Z}$ is the set of integers. $\mathbf{Z}_{+}:=$ $\{j \in \mathbf{Z} \mid j \geq 0\} . \mathbf{Z}_{-}:=\{j \in \mathbf{Z} \mid j<0\}$. The unit circle of the complex plane is $\mathbf{T}$, and $\mathbf{D}$ is the open unit disk. If $H$ is a Hilbert space, then $\mathcal{L}(H)$ denotes the bounded linear operators in $H$. Elements of a Hilbert space are denoted by lower case letters; for example $u \in U$. Sequences in Hilbert spaces are denoted by $\tilde{u}=\left\{u_{i}\right\}_{i \in I} \subset U$, where $I$ is the index set. Usually $I=\mathbf{Z}$ or $I=\mathbf{Z}_{+}$. Given a Hilbert space $Z$, we define the sequence spaces

$$
\begin{aligned}
\operatorname{Seq}(Z) & :=\left\{\left\{z_{i}\right\}_{i \in \mathbf{Z}}: z_{i} \in Z \text { and } \exists I \in \mathbf{Z} \quad \forall i \leq I: z_{i}=0\right\} \\
\operatorname{Seq}_{+}(Z) & :=\left\{\left\{z_{i}\right\}_{i \in \mathbf{Z}}: z_{i} \in Z \text { and } \forall i<0: z_{i}=0\right\} \\
\operatorname{Seq}_{-}(Z) & :=\left\{\left\{z_{i}\right\}_{i \in \mathbf{Z}} \in \operatorname{Seq}(Z): z_{i} \in Z \quad \text { and } \quad \forall i \geq 0: z_{i}=0\right\} \\
\ell^{2}(\mathbf{Z} ; Z) & :=\left\{\left\{z_{i}\right\}_{i \in I} \subset Z: \sum_{i \in I}\left\|z_{i}\right\|_{Z}^{2}<\infty\right\}, \quad \text { where } I=\mathbf{Z}, \mathbf{Z}_{+}, \quad \text { or } \quad \mathbf{Z}_{-},
\end{aligned}
$$


where the last are Hilbert spaces with obvious inner products. The following linear operators are defined in $\operatorname{Seq}(Z)$ and $\ell^{2}(\mathbf{Z} ; Z)$ :

- the interval projections for $j, k \in \mathbf{Z}$

$$
\begin{aligned}
& \pi_{[j, k]} \tilde{z}:=\left\{w_{j}\right\} ; \quad w_{i}=z_{i} \quad \text { for } \quad j \leq i \leq k, \quad 0 \quad \text { otherwise; } \\
& \pi_{j}:=\pi_{[j, j]},
\end{aligned}
$$

- the future and past projections

$$
\pi_{+}:=\pi_{[1, \infty]}, \quad \pi_{-}:=\pi_{[-\infty,-1]},
$$

- the composite projections

$$
\bar{\pi}_{+}:=\pi_{0}+\pi_{+}, \quad \bar{\pi}_{-}:=\pi_{0}+\pi_{-},
$$

- the bilateral forward time shift $\tau$ and its (formal) adjoint, then backward time shift $\tau^{*}$

$$
\begin{aligned}
& \tau \tilde{u}:=\left\{w_{j}\right\} \quad \text { where } \quad w_{j}=u_{j-1} \\
& \tau^{*} \tilde{u}:=\left\{w_{j}\right\} \quad \text { where } \quad w_{j}=u_{j+1} .
\end{aligned}
$$

The above projections are orthogonal in $\ell^{2}(\mathbf{Z} ; Z)$. The bilateral shift $\tau$ is unitary in $\ell^{2}(\mathbf{Z} ; Z)$. The following identifications are used throughout this paper: $\ell^{2}\left(\mathbf{Z}_{+} ; Z\right)=$ $\bar{\pi}_{+} \ell^{2}(\mathbf{Z} ; Z), \ell^{2}\left(\mathbf{Z}_{-} ; Z\right)=\pi_{-} \ell^{2}(\mathbf{Z} ; Z) . \quad Z=\pi_{j} \ell^{2}(\mathbf{Z} ; Z)$ for $j \in \mathbf{Z}$. Other notations are introduced when they are needed.

Our basic setting is a fixed realization of the transfer function that is neither assumed to be input nor output stable. The realization we are working with is regarded as the given data, no matter how (topologically) uncomfortable it is; i.e. we work with the given operators in the original topologies. We call this realization a discrete time linear system (DLS). It is given by a system of difference equations

$$
\left\{\begin{array}{l}
x_{j+1}=A x_{j}+B u_{j}, \\
y_{j}=C x_{j}+D u_{j}, \quad j \geq 0,
\end{array}\right.
$$

where $u_{j} \in U, x_{j} \in H, y_{j} \in Y$, and $A, B, C$ and $D$ are bounded linear operators between appropriate Hilbert spaces. We call the ordered quadruple $\phi=(\underset{C}{A} \underset{D}{B})$ a $D L S$ in difference equation form. The three Hilbert spaces are as follows: $U$ is the input space, $H$ is the state space and $Y$ is the output space of $\phi$.

There is also another equivalent form for DLS, called DLS in I/O-form (see [9, Theorem 11]). It consists of four linear operators in the ordered quadruple

$$
\Phi:=\left[\begin{array}{cc}
A^{j} & \mathcal{B} \tau^{* j} \\
\mathcal{C} & \mathcal{D}
\end{array}\right]
$$


Note that $\phi$ stands for the DLS in difference equation form, and the capital $\Phi$ is the same DLS written in I/O-form. The operator $A \in \mathcal{L}(H)$ is called the semi-group generator, and the family $\left\{A^{j}\right\}_{j \geq 0}$ is called the semi-group of $\Phi$. It is the same operator $A$ that appears in the corresponding DLS $\phi$ in difference equation form. $\mathcal{B}: S e q_{-}(U) \rightarrow H$ is called the controllability map that maps the past input into present state. $\mathcal{C}: H \rightarrow \operatorname{Seq}_{+}(Y)$ is called the observability map that maps the present state into future outputs. The operator $\mathcal{D}: S e q(U) \rightarrow \operatorname{Seq}(Y)$ in (2) is called the I/O-map that maps the input into output in a causal and shift invariant way. The operators in $\Phi$ and $\phi$ are connected by straightforward algebraic relations (see [9, Lemma 7 and Definition 9]):

- $\mathcal{B}: \operatorname{Seq}_{-}(U) \rightarrow H, \mathcal{C}: H \rightarrow \operatorname{Seq}_{+}(Y)$ and $\mathcal{D}: \operatorname{Seq}(U) \rightarrow \operatorname{Seq}(Y)$.

- $\mathcal{D}, \mathcal{B}$ and $\mathcal{C}$ are causal; i.e. they satisfy

$$
\pi_{-} \mathcal{D} \bar{\pi}_{+}=0, \quad \mathcal{B} \bar{\pi}_{+}=0, \quad \pi_{-} \mathcal{C}=0
$$

- $\mathcal{B}$ satisfies

$$
\begin{aligned}
& \mathcal{B} \tau^{*}=A \mathcal{B}+\mathcal{B} \tau^{*} \pi_{0}, \\
& \mathcal{B} \tau^{* j} \tilde{u}=A^{j} \mathcal{B} \tilde{u}+\sum_{i=0}^{j-1} A^{i} B u_{j-i-1}, \\
& B=\mathcal{B} \pi_{-1} \in \mathcal{L}(U, H),
\end{aligned}
$$

where $U$ is identified with range $\left(\pi_{-1}\right)$ on $\operatorname{Seq}(U)$ in the natural way.

- $\mathcal{C}$ satisfies

$$
\begin{aligned}
& \bar{\pi}_{+} \tau^{*} \mathcal{C}=\mathcal{C} A, \\
& C=\pi_{0} \mathcal{C} \in \mathcal{L}(H, Y),
\end{aligned}
$$

where $Y$ is identified with range $\left(\pi_{0}\right)$ on $\operatorname{Seq}(Y)$ in the natural way.

- $\mathcal{D}$ satisfies

$$
\begin{aligned}
& \bar{\pi}_{+} \mathcal{D} \pi_{-}=\mathcal{C B}, \\
& \mathcal{D} \tau=\tau \mathcal{D}, \quad \mathcal{D} \tau^{*}=\tau^{*} \mathcal{D} \\
& D=\pi_{0} \mathcal{D} \pi_{0} \in \mathcal{L}(U, Y)
\end{aligned}
$$

where $U, Y$ are identified with range $\left(\pi_{0}\right)$ in the natural way.

For the input, output and state sequences the following notation is used:

- The state of $\phi$ at time $j \geq 0$ is denoted by $x_{j}\left(x_{0}, \tilde{u}\right)$, and it is defined by

$$
x_{j}\left(x_{0}, \tilde{u}\right):=A^{j} x_{0}+\sum_{i=0}^{j-1} A^{i} B u_{j-i}=A^{j} x_{0}+\mathcal{B}_{\phi} \tau^{* j} \tilde{u} .
$$


- The output sequence $\tilde{y}\left(x_{0}, \tilde{u}\right):=\left\{y_{j}\left(x_{0}, \tilde{u}\right)\right\}_{j \in \mathbf{Z}_{+}}$of $\phi$ is defined by

$$
y_{j}\left(x_{0}, \tilde{u}\right):=C A^{j} x_{0}+\sum_{i=0}^{j-1} C A^{i} B u_{j-i}+D u_{j}=\pi_{j}\left(\mathcal{C}_{\phi} x_{0}+\mathcal{D}_{\phi} \tilde{u}\right),
$$

where $x_{0} \in H$ denotes the initial state at time $j=0$, and $\tilde{u} \in S e q_{+}(U)$ is an input sequence.

In this paper our main emphasis is upon I/O-stable DLS's; this means that the Toeplitz operator $\mathcal{D} \bar{\pi}_{+}: \ell^{2}\left(\mathbf{Z}_{+} ; U\right) \rightarrow \ell^{2}\left(\mathbf{Z}_{+} ; Y\right)$ is a bounded. Then the Toeplitz operator has a bounded extension to the whole of $\ell^{2}(\mathbf{Z} ; U)$, also denoted by $\mathcal{D}$. In the frequency domain, the action of $\mathcal{D}$ is the multiplication by the $H^{\infty}$-transfer function of the system.

For the study of the operators $\mathcal{B}$ and $\mathcal{C}$, a suitable definition is needed for their domains $\left(\left[9\right.\right.$, Definition 24]). We define $\operatorname{dom}(\mathcal{B}):=S e q_{-}(U)$, equipped with the $\ell^{2}(\mathbf{Z} ; U)$-inner product. The domain of $\mathcal{C}$ is given by

$$
\operatorname{dom}(\mathcal{C}):=\left\{x_{0} \in H \mid \mathcal{C} x_{0} \in \ell^{2}\left(\mathbf{Z}_{+} ; Y\right)\right\}
$$

equipped with the inner product topology of $H$. Neither of the operators $\mathcal{B}, \mathcal{C}$ are assumed to be bounded in their domains, but $\mathcal{C}$ is closed (see [9, Lemma 27]). If they are bounded, we say that $\Phi$ is input stable or output stable, respectively.

The stability notions associated to the semi-group generator $A$ of the DLS $\Phi$ are the following (see [9, Definition 21])

- $A$ is power (or exponentially) stable, if $\rho(A)<1$,

- $A$ is strongly stable, if $A^{j} x_{0} \rightarrow 0$ as $j \rightarrow \infty$,

- $A$ is power bounded, if $\sup _{j \geq 0}\left\|A^{j}\right\|_{H}<\infty$.

We say that $\Phi$ is stable if it is I/O-stable, input stable, output stable and its $A$ semigroup generator is power bounded. If $\Phi$ is stable and $A$ is strongly stable, then $\Phi$ is strongly stable. The relations between various stability condition are discussed in $[9$, Section $6]$. We note that the I/O-stability implies that range $(\mathcal{B}) \subset \operatorname{dom}(\mathcal{C})$; this is known as the compatibility condition in [9, Lemma 39]). We assume throughout this paper that $\overline{\operatorname{dom}(\mathcal{C})}=H$. In Lemma 39 and Theorem 40 we assume further that $\overline{\text { range }(\mathcal{B})}=H$.

The notion of state feedback is central in this work. In difference equation form, we realize the state feedback by first adding still another equation $u_{j}=K x_{j}+F u_{j}$ to equations (1), where $K \in \mathcal{L}(U)$. This gives us an extended $D L S \phi^{\text {ext }}$. We get the closed loop DLS $\phi_{\diamond}^{\text {ext }}$ in difference equation form by simple manipulation. However, in this paper we need the same structure written in I/O-form.

In I/O-form, the new output signal given by $K$ provides a new output $\tilde{v} \in \ell^{2}\left(\mathbf{Z}_{+} ; U\right)$ to $\Phi$, thus giving an (open loop) extended $D L S \Phi^{\text {ext }}:=[\Phi,[\mathcal{K}, \mathcal{F}]]$. This is a cartesian product 
of two DLS's with the same input and semi-group structure, as presented in the following picture:

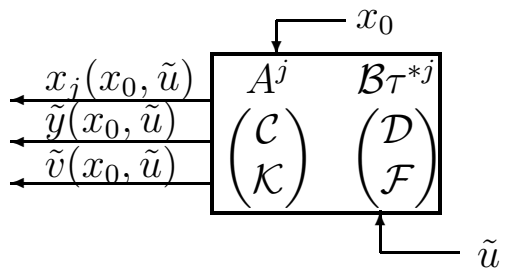

The ordered pair of operators $[\mathcal{K}, \mathcal{F}]$ is called a feedback pair of $\Phi$. Here $\mathcal{K}$ is a valid observability map and $\mathcal{F}$ is a valid I/O-map for the system with semi-group generator $A$ and controllability map $\mathcal{B}$; the operator $(\mathcal{I}-\mathcal{F})^{-1}: S e q(U) \rightarrow S e q(U)$ is required to be causal and shift invariant. From an $I / O$-stable feedback pair we require that $\operatorname{dom}(\mathcal{C}) \subset$ $\operatorname{dom}(\mathcal{K})$, and both $\mathcal{F}$ and $(\mathcal{I}-\mathcal{F})^{-1}$ are bounded in the $\ell^{2}$-topology. If, in addition, $\mathcal{K}: H \rightarrow \ell^{2}\left(\mathbf{Z}_{+} ; U\right)$ is bounded, then we say that $[\mathcal{K}, \mathcal{F}]$ is stable. The closed loop extended $D L S \Phi_{\diamond}^{e x t}$ is the DLS that we obtain when we close the following state feedback connection:

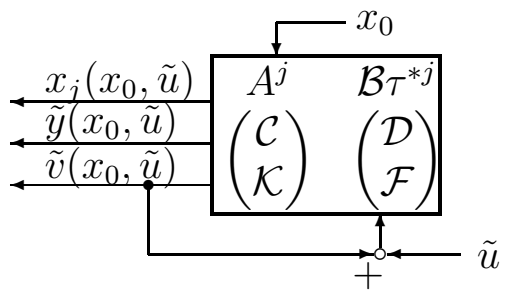

The formulae for the closed loop system in terms of the open loop operators can be easily calculated (see [9, Definition 18]). Thus we have two different notions of state feedback; one for DLS's in difference equation form, the other for DLS's in I/O-form. It follows that these feedback notions are equivalent in the same way than the two notions of the DLS are equivalent (see [9, Section 5]). The stability properties of the open and closed loop feedback systems are discussed in [9, Section 9].

We remark that the structure described above is closely related to the concept of a (continuous time) stable well-posed linear system in [19], [27] and [28]. The notation of this paper and [9] is a discrete time variant of that used in the continuous time papers [19], [21] and [20].

The introduction of two different but equivalent forms of DLS's may first seem superfluouseven more so because of the fact that the I/O -stable $\left(H^{\infty}\right)$ systems we can use the transfer function representation (see [17, Theorem 1.15B]). However, operator theoretic study of these systems become notationally very clumsy, if the basic operators are always stated as multiplications by transfer functions. We remark that in [17] the basic objects are unilateral shift operators together with Toeplitz operators, and the complex analysis results are presented more or less as an important application. From the control theoretic point of view, the interaction between controllability, observability and I/O -maps can be conveniently described in our formalism because these operators are the basic building blocks of the DLS in I/O -form. Also the generalizations to non-linear theories can be done easily with this notation. 


\section{Nonstandard cost and minimax control of DLS's}

We consider a minimax control problem associated to a DLS $\Phi=\left[\begin{array}{cc}A^{j} & \mathcal{B}_{\tau^{* j}} \\ \mathcal{C} & \mathcal{D}\end{array}\right]$ and a possibly non-definite cost functional measuring the outputs of $\Phi$. Basic definitions are given and facts proved in this section.

We start with picking a self-adjoint operator $J \in \mathcal{L}(Y)$ which induces a nonstandard (i.e. not necessarily positive definite) inner product on the output space of $\Phi$. The cost functional is defined as follows:

Definition 1. Let $\Phi=\left[\begin{array}{cc}A_{\mathcal{C}}^{j} & \mathcal{B} \tau^{* j} \\ \mathcal{D}\end{array}\right]$ be a $D L S$, and let $J \in \mathcal{L}(Y), R \in \mathcal{L}(U)$ be self-adjoint. Then the nonstandard cost for the output $\tilde{y}$ of $\Phi$ is

$$
J\left(x_{0}, \tilde{u}\right):=\sum_{j \geq 0}\left[\left(y_{j}\left(x_{0}, \tilde{u}\right), J y_{j}\left(x_{0}, \tilde{u}\right)\right)_{Y}+\left(u_{j}, R u_{j}\right)_{U}\right]
$$

where $\tilde{u} \in \ell^{2}\left(\mathbf{Z}_{+} ; U\right)$ is an input and $x_{0} \in \operatorname{dom}(\mathcal{C})$ is the initial state of the system at time $j=0$.

It is a known fact that the control $\tilde{u}$ can always be thought to be "free of charge" (no cost on the input), because the input can be made visible in the output. Then the cost for the control can always be included in the cost for the output. Technically this is accomplished by replacing the DLS $\left(\begin{array}{cc}A & B \\ D\end{array}\right)$ by an extended system $\phi^{\prime}=\left(\begin{array}{cc}A & B \\ C^{\prime} & D^{\prime}\end{array}\right)$, where $C^{\prime} \in \mathcal{L}(U, Y \times U)$, $D^{\prime} \in \mathcal{L}(H, Y \times U)$, and $J$ by $J^{\prime} \in \mathcal{L}(Y \times U, Y \times U)$ defined by

$$
C^{\prime}=\left(\begin{array}{c}
C \\
0
\end{array}\right), \quad D^{\prime}=\left(\begin{array}{c}
D \\
I
\end{array}\right), \quad J^{\prime}=\left(\begin{array}{cc}
J & 0 \\
0 & R
\end{array}\right)
$$

Then, if $z_{k}\left(x_{0}, \tilde{u}\right):=C^{\prime} x_{k}+D^{\prime} u_{k}$ is the output of $\Phi^{\prime}$, we get

$$
\left(y_{j}\left(x_{0}, \tilde{u}\right), J y_{j}\left(x_{0}, \tilde{u}\right)\right)_{Y}+\left(u_{j}, R u_{j}\right)_{U}=\left(z_{k}\left(x_{0}, \tilde{u}\right), J^{\prime} z_{k}\left(x_{0}, \tilde{u}\right)\right)_{Y \times X} .
$$

Thus there is no loss of generality in setting $R=0$ in formula (6), and this is what we always do. In this case equation (6) takes the form

$$
J\left(x_{0}, \tilde{u}\right)=\left\langle\mathcal{C} x_{0}+\mathcal{D} \tilde{u}, J\left(\mathcal{C} x_{0}+\mathcal{D} \tilde{u}\right)\right\rangle_{\ell^{2}\left(\mathbf{z}_{+} ; Y\right)} .
$$

Note that we use the same letter $J$ for both the self-adjoint operator and for the associated cost functional. To avoid trivialities, we see that the inner product in equation (8) is finite for those $x_{0}$ and $\tilde{u}$ that we use.

Proposition 2. Let $J \in \mathcal{L}(Y)$ and $\Phi$ be an I/O-stable DLS. Then $\left|J\left(x_{0}, \tilde{u}\right)\right|<\infty$ for all $x_{0} \in \operatorname{dom}(\mathcal{C})$ and $\tilde{u} \in \ell^{2}\left(\mathbf{Z}_{+} ; U\right)$.

Proof. If $x_{0} \in \operatorname{dom}(\mathcal{C})$ and $\tilde{u} \in \ell^{2}\left(\mathbf{Z}_{+} ; U\right)$, then by the definition of $\operatorname{dom}(\mathcal{C})$ and I/Ostability, $\mathcal{C} x_{0}+\mathcal{D} \tilde{u} \in \ell^{2}\left(\mathbf{Z}_{+} ; Y\right)$. The claim immediately follows. 
If $J$ is positive, then one would immediately be tempted to find the optimal control that minimizes the cost. With the nonstandard case, the cost could be made as large or small as we please, just by choosing a suitable input $\tilde{u}$. So there is not much sense in speaking about minimal or maximal cost. We look for certain control sequences, called critical controls $\tilde{u}^{\text {crit }}\left(x_{0}\right)$, that are saddle points of the cost functional $J\left(x_{0}, \tilde{u}\right)$ as a mapping from $\ell^{2}\left(\mathbf{Z}_{+} ; U\right)$ onto $\mathbf{R}$.

Definition 3. Let $\Phi=\left[\begin{array}{cc}A^{j} & \mathcal{B} \tau^{* j} \\ \mathcal{D} & \mathcal{D}\end{array}\right]$ be a DLS, and let $x_{0} \in \operatorname{dom}(\mathcal{C})$ be an initial state.

(i) The control $\tilde{u}^{\text {crit }}\left(x_{0}\right) \in S e q_{+}(U)$ is critical if the Frechet derivative of the cost $J\left(x_{0}, \tilde{u}\right)$ with respect to $\tilde{u}$ vanishes.

(ii) The corresponding critical state sequence $\left\{x_{j}^{\text {crit }}\left(x_{0}\right)\right\}_{j \geq 0}$ is defined by

$$
x_{j}^{\text {crit }}\left(x_{0}\right)=x_{j}\left(x_{0}, \tilde{u}^{\text {crit }}\left(x_{0}\right)\right) .
$$

(iii) The corresponding critical output $\tilde{y}^{\text {crit }}\left(x_{0}\right)$ is defined by

$$
\tilde{y}^{\text {crit }}\left(x_{0}\right)=\mathcal{C} x_{0}+\mathcal{D} \tilde{u}^{\text {crit }}\left(x_{0}\right) \text {. }
$$

Let us first calculate a necessary and sufficient condition for a control to be critical, without worrying about existence and uniqueness questions of the critical control.

Lemma 4. Let $\Phi=\left[\begin{array}{cc}A^{j} & \mathcal{B}_{\mathcal{C}}^{* j} \\ \mathcal{C} & \mathcal{D}\end{array}\right]$ be an I/O-stable DLS, and let $x_{0} \in \operatorname{dom}(\mathcal{C})$ be an initial state. Then the control $\tilde{u}^{\text {crit }}\left(x_{0}\right) \in \ell^{2}\left(\mathbf{Z}_{+}, U\right)$ is critical if and only if

$$
\bar{\pi}_{+} \mathcal{D}^{*} J \mathcal{C} x_{0}=-\bar{\pi}_{+} \mathcal{D}^{*} J \mathcal{D} \tilde{u}^{\text {crit }}\left(x_{0}\right) \text {. }
$$

Furthermore, the corresponding critical output $\tilde{y}^{\text {crit }}\left(x_{0}\right)$ satisfies

$$
\bar{\pi}_{+} \mathcal{D}^{*} J \tilde{y}^{\text {crit }}\left(x_{0}\right)=0 .
$$

Proof. We have for $\tilde{u} \in \ell^{2}\left(Z_{+} ; U\right)$

$$
J\left(x_{0}, \tilde{u}\right)=\left\langle\mathcal{C} x_{0}+\mathcal{D} \tilde{u}, J\left(\mathcal{C} x_{0}+\mathcal{D} \tilde{u}\right)\right\rangle_{\ell^{2}\left(\mathbf{z}_{+} ; Y\right)} .
$$

The critical control is found by requiring the real derivative $\frac{d}{d \epsilon} J\left(x_{0}, \tilde{u}+\epsilon \tilde{w}\right)=0$ at $\epsilon=0$ for all $\tilde{w} \in \ell^{2}\left(Z_{+} ; U\right)$. This gives

$$
\begin{aligned}
& \left.\frac{d}{d \epsilon} J\left(x_{0}, \tilde{u}+\epsilon \tilde{w}\right)\right|_{\epsilon=0} \\
& =2 \operatorname{Re}\left\langle\tilde{w}, \bar{\pi}_{+} \mathcal{D}^{*} J \mathcal{C} x_{0}+\bar{\pi}_{+} \mathcal{D}^{*} J \tilde{\mathcal{D}} \tilde{u}^{c r i t}\left(x_{0}\right)\right\rangle_{\ell^{2}\left(\mathbf{Z}_{+} ; Y\right)}=0,
\end{aligned}
$$

which gives equations (9) and (10).

The Toeplitz operator $\bar{\pi}_{+} \mathcal{D}^{*} J \mathcal{D} \bar{\pi}_{+}$is called the Popov operator (see [6]) or the power spectrum operator (see [5]) of the DLS. The following definition gives us the basic notion of this paper, namely $J$-coercivity. It serves as a sufficient condition for the existence of the unique control. 
Definition 5. The DLS $\Phi=\left[\begin{array}{cc}A^{j} & \mathcal{B}_{\tau^{* j}} \\ \mathcal{C} & \mathcal{D}\end{array}\right]$ is $J$-coercive, if the Toeplitz operator $\bar{\pi}_{+} \mathcal{D}^{*} J \mathcal{D} \bar{\pi}_{+}$has a bounded inverse in $\ell^{2}\left(\mathbf{Z}_{+} ; U\right)$.

Proposition 6. Let $\Phi$ be an I/O-stable and J-coercive DLS. Then $\mathcal{D} \bar{\pi}_{+}$is coercive. In particular, range $\left(\mathcal{D} \bar{\pi}_{+}\right)$is closed.

Proof. To show coercivity, assume for contradiction that there is a sequence $\left\{\tilde{u}_{j}\right\} \subset$ $\ell^{2}\left(\mathbf{Z}_{+} ; U\right),\left\|\tilde{u}_{j}\right\|_{\ell^{2}\left(\mathbf{Z}_{+} ; U\right)}=1$ such that $\mathcal{D} \bar{\pi}_{+} \tilde{u}_{j} \rightarrow 0$ as $j \rightarrow 0$. Because $\mathcal{D}$ is bounded by I/O-stability, so is $\bar{\pi}_{+} \mathcal{D}^{*} J$. But then $\bar{\pi}_{+} \mathcal{D}^{*} J \mathcal{D} \bar{\pi}_{+} \tilde{u}_{j} \rightarrow 0$ as $j \rightarrow 0$. This is a contradiction against the $J$-coercivity of $\Phi$.

Now equation (9) immediately calls for the following definition and lemma:

Definition 7. Let $\Phi=\left[\begin{array}{cc}A_{\mathcal{C}}^{j} & \mathcal{B} \tau^{* j} \\ \mathcal{D}\end{array}\right]$ be an I/O-stable and J-coercive DLS. Then

(i) the the densely defined linear operator $\mathcal{K}^{\text {crit }}: H \supset \operatorname{dom}\left(\mathcal{K}^{\text {crit }}\right) \rightarrow \ell^{2}\left(\mathbf{Z}_{+} ; U\right)$, defined by

$$
\mathcal{K}^{c r i t}:=-\left(\bar{\pi}_{+} \mathcal{D}^{*} J \mathcal{D} \bar{\pi}_{+}\right)^{-1} \bar{\pi}_{+} \mathcal{D}^{*} J \mathcal{C}
$$

is called the critical (closed loop) feedback operator, where dom $\left(\mathcal{K}^{\text {crit }}\right):=\left\{x_{0} \in\right.$ $\left.H \mid \mathcal{K}^{c r i t} x_{0} \in \ell^{2}\left(\mathbf{Z}_{+} ; Y\right)\right\}$

(ii) the the densely defined linear operator $K^{\text {crit }}: H \supset \operatorname{dom}\left(K^{\text {crit }}\right) \rightarrow \ell^{2}\left(\mathbf{Z}_{+} ; U\right)$, defined by

$$
K^{\text {crit }}:=\pi_{0} \mathcal{K}^{\text {crit }}
$$

(the spaces range $\left(\pi_{0}\right)$ and $U$ have been identified) is called the critical (closed loop) one step feedback operator, where $\operatorname{dom}\left(K^{\text {crit }}\right):=\operatorname{dom}\left(\mathcal{K}^{\text {crit }}\right)$,

(iii) the densely defined linear operator $\mathcal{C}^{\text {crit }}: H \supset \operatorname{dom}\left(\mathcal{C}^{\text {crit }}\right) \rightarrow \ell^{2}\left(\mathbf{Z}_{+} ; Y\right)$, defined by

$$
\mathcal{C}^{\text {crit }}:=\mathcal{C}+\mathcal{D} \mathcal{K}^{\text {crit }}
$$

is called the critical (closed loop) observability map, where $\operatorname{dom}\left(\mathcal{C}^{\text {crit }}\right):=\left\{x_{0} \in\right.$ $\left.H \mid \mathcal{C}^{\text {crit }} x_{0} \in \ell^{2}\left(\mathbf{Z}_{+} ; Y\right)\right\}$.

It is easy to see that the above operators are well defined in their domains. This requires checking that all the presented operator products make sense. For I/O-stable and $J_{-}$ coercive DLS's, clearly $\operatorname{dom}(\mathcal{C}) \subset \operatorname{dom}\left(\mathcal{K}^{\text {crit }}\right)$ and $\operatorname{dom}(\mathcal{C}) \subset \operatorname{dom}\left(\mathcal{C}^{\text {crit }}\right)$. If $K^{\text {crit }}$ is bounded, we can identify it with its continuous extension to the whole of $H$. By a simple manipulation, we see that

$$
\mathcal{C}^{\text {crit }}=\left(\bar{\pi}_{+}-\bar{\pi}_{+} \mathcal{D}\left(\bar{\pi}_{+} \mathcal{D}^{*} J \mathcal{D} \bar{\pi}_{+}\right)^{-1} \bar{\pi}_{+} \mathcal{D}^{*} J\right) \mathcal{C}=: \Pi \mathcal{C},
$$

where $\Pi$ is a bounded projection (by I/O-stability and $J$-coercivity) in $\ell^{2}\left(\mathbf{Z}_{+} ; U\right)$ commuting with $J$. 
Lemma 8. Assume that the $D L S \Phi=\left[\begin{array}{cc}A^{j} & \mathcal{B}_{\tau^{* j}} \\ \mathcal{C} & \mathcal{D}\end{array}\right]$ is I/O-stable and J-coercive. Then

(i) for each $x_{0} \in \operatorname{dom}(\mathcal{C})$ there is a unique critical control $\tilde{u}^{\text {crit }}\left(x_{0}\right)$ satisfying formula (9),

(ii) the critical control satisfies

$$
\tilde{u}^{\text {crit }}\left(x_{0}\right)=\mathcal{K}^{c r i t} x_{0}
$$

the critical output satisfies

$$
\tilde{y}^{\text {crit }}\left(x_{0}\right)=\mathcal{C}^{\text {crit }} x_{0}
$$

and the critical trajectory satisfies

$$
x_{j}^{c r i t}\left(x_{0}\right)=A^{\text {crit }}(j) x_{0} .
$$

Proof. Use Definitions 5, 7, Lemma 4 and basic properties of DLS's.

The family of operators $\left.\left\{A^{\text {crit }}(j)\right\}_{j \geq 0}\right)$ is in fact a semi-group of linear operators defined in $\operatorname{dom}(\mathcal{C})$. This is the subject of the following lemma.

Lemma 9. Assume that the $D L S \Phi=\left[\begin{array}{cc}A_{\mathcal{C}}^{j} & \mathcal{B}_{\mathcal{D}}^{* j}\end{array}\right]$ is I/O-stable and J-coercive. Then

(i) the linear operators $A^{\text {crit }}(j):=A^{j}+\mathcal{C} \tau^{* j} \mathcal{K}^{\text {crit }}: \operatorname{dom}(\mathcal{C}) \rightarrow H$ for $j \geq 1$ satisfy

$$
A^{\text {crit }}(j) \operatorname{dom}(\mathcal{C}) \subset \operatorname{dom}(\mathcal{C}),
$$

(ii) the family $\left\{A^{\text {crit }}(j)\right\}_{j \geq 0}$ of linear operators defined in $\operatorname{dom}(\mathcal{C})$ is a semi-group

$$
A^{\text {crit }}(j)=\left(A^{\text {crit }}\right)^{j}
$$

for all $j \in \mathbf{Z}_{+}$, where $A^{\text {crit }}:=A^{\text {crit }}(1)$ is a linear operator on $\operatorname{dom}(\mathcal{C})$, called the critical semi-group generator,

(iii) the critical trajectory $\left\{x_{j}^{\text {crit }}\left(x_{0}\right)\right\}_{j \geq 0}$ associated to the initial value $x_{0} \in \operatorname{dom}(\mathcal{C})$ is given by

$$
x_{j}^{\text {crit }}\left(x_{0}\right)=\left(A^{\text {crit }}\right)^{j} x_{0}
$$

Proof. The proof of claim (i) is a consequence of the fact that $\Phi$, as an I/O-stable system, satisfies range $(\mathcal{B}) \subset \operatorname{dom}(\mathcal{C})\left(\right.$ see $\left[9\right.$, Lemma 40] ). Because always $\pi_{-} \tau^{* j} \mathcal{K}^{\text {crit }} x_{0} \in$ $\operatorname{dom}(\mathcal{B})$ by the definition of $\operatorname{dom}(\mathcal{B})$, claim (i) immediately follows.

To prove (ii) we use a same kind of approach as in the proof of Lemma 4. Fix $x_{0} \in$ $\operatorname{dom}\left(\mathcal{K}^{\text {crit }}\right)=\operatorname{dom}(\mathcal{C}), j \geq 1$. Let $\epsilon>0$ and $\tilde{w} \in \ell^{2}\left(\mathbf{Z}_{+} ; U\right)$ be arbitrary. Then we have

$$
\begin{aligned}
& J\left(x_{0}, \tilde{u}^{c r i t}\left(x_{0}\right)+\epsilon \tau^{j} \tilde{w}\right) \\
& =\left\langle\pi_{[0, j-1]}\left[\mathcal{C} x_{0}+\mathcal{D} \tilde{u}^{c r i t}\left(x_{0}\right)\right], J(-,,-)\right\rangle_{\ell^{2}\left(\mathbf{z}_{+} ; Y\right)} \\
& +\left\langle\pi_{[j, \infty]}\left[\mathcal{C} x_{0}+\mathcal{D}\left(\tilde{u}^{c r i t}\left(x_{0}\right)+\epsilon \tau^{j} \tilde{w}\right)\right], J(-,,-)\right\rangle_{\ell^{2}\left(\mathbf{Z}_{+} ; Y\right)},
\end{aligned}
$$


because $\pi_{[0, j-1]} \mathcal{D}\left(\epsilon \tau^{j} \tilde{w}\right)=\pi_{[0, j-1]} \tau^{j}(\mathcal{D}(\epsilon \tilde{w}))=0$ as a consequence of the causality of $\mathcal{D}$. A simple calculation, together with Definition 7 , allows us to continue

$$
\begin{aligned}
& J\left(x_{0}, \tilde{u}^{c r i t}\left(x_{0}\right)+\epsilon \tau^{j} \tilde{w}\right) \\
& =\left\langle\pi_{[0, j-1]} \mathcal{C}^{c r i t} x_{0}, J(-,,-)\right\rangle_{\ell^{2}\left(\mathbf{Z}_{+} ; Y\right)}+\left\langle\pi_{[j, \infty]}\left[\mathcal{C}^{c r i t} x_{0}+\epsilon \tau^{j} \mathcal{D} \tilde{w}\right], J(-,,-)\right\rangle_{\ell^{2}\left(\mathbf{Z}_{+} ; Y\right)} \\
& =\left\langle\mathcal{C}^{c r i t} x_{0}, J \mathcal{C}^{c r i t}\right\rangle_{\ell^{2}\left(\mathbf{Z}_{+} ; Y\right)}+2 \epsilon \operatorname{Re}\left\langle\pi_{[j, \infty]} \mathcal{C}^{c r i t} x_{0}, J \tau^{j} \mathcal{D} \tilde{w}\right\rangle_{\ell^{2}\left(\mathbf{Z}_{+} ; Y\right)} \\
& +\epsilon^{2}\left\langle\mathcal{D}^{*} J \mathcal{D} \tilde{w}, \tilde{w}\right\rangle_{\ell^{2}\left(\mathbf{Z}_{+} ; Y\right)}
\end{aligned}
$$

Now because $\tilde{u}^{\text {crit }}\left(x_{0}\right)$ is critical, we must have $\left.\frac{d}{d \epsilon} J\left(x_{0}, \tilde{u}^{\text {crit }}\left(x_{0}\right)+\epsilon \tau^{j} \tilde{w}\right)\right)=0$ at $\epsilon=0$ for all $\tilde{w} \in \ell^{2}(\mathbf{Z} ; U), j \geq 0$. It follows that $\operatorname{Re}\left\langle\pi_{[j, \infty]} \mathcal{C}^{\text {crit }} x_{0}, J \tau^{j} \mathcal{D} \tilde{w}\right\rangle_{\ell^{2}\left(\mathbf{Z}_{+} ; Y\right)}=0$ for all $\tilde{w}$, and then immediately for all $j \geq 0$

$$
\bar{\pi}_{+} \mathcal{D}^{*} J \bar{\pi}_{+} \tau^{* j} \mathcal{C}^{c r i t} x_{0}=\bar{\pi}_{+} \mathcal{D}^{*} J \bar{\pi}_{+} \tau^{* j}\left(\mathcal{C}+\mathcal{D} \mathcal{K}^{c r i t}\right) x_{0}=0
$$

and

$$
\begin{aligned}
& \bar{\pi}_{+} \mathcal{D}^{*} J \bar{\pi}_{+} \tau^{* j} \mathcal{C} x_{0}=\bar{\pi}_{+} \mathcal{D}^{*} J \mathcal{C} A^{j} x_{0}=-\bar{\pi}_{+} \mathcal{D}^{*} J \bar{\pi}_{+} \mathcal{D} \tau^{* j} \mathcal{K}^{c r i t} x_{0} \\
& =-\left(\bar{\pi}_{+} \mathcal{D}^{*} J \mathcal{D} \bar{\pi}_{+}\right) \tau^{* j} \mathcal{K}^{c r i t} x_{0}-\bar{\pi}_{+} \mathcal{D}^{*} J\left(\bar{\pi}_{+} \mathcal{D} \pi_{+}\right) \tau^{* j} \mathcal{K}^{c r i t} x_{0}
\end{aligned}
$$

Using $\bar{\pi}_{+} \mathcal{D} \pi_{-}=\mathcal{C B}$, gives $\bar{\pi}_{+} \mathcal{D}^{*} J \mathcal{C}\left(A^{j}+\mathcal{B} \tau^{* j} \mathcal{K}^{c r i t}\right) x_{0}=-\left(\bar{\pi}_{+} \mathcal{D}^{*} J \mathcal{D} \bar{\pi}_{+}\right) \tau^{* j} \mathcal{K}^{c r i t} x_{0}$ for $x_{0} \in \operatorname{dom}(\mathcal{C}) j \geq 1$. This implies by Definition 7

$$
\bar{\pi}_{+} \tau^{* j} \mathcal{K}^{c r i t} x_{0}=\mathcal{K}^{c r i t} A^{c r i t}(j) x_{0} .
$$

The rest of the proof is now a calculation. For $k \geq 0, j \geq 1$ we have by Lemma 8

$$
\begin{aligned}
& A^{\text {crit }}(k) A^{\text {crit }}(j) x_{0}=A^{k} x_{j}^{c r i t}\left(x_{0}\right)+\mathcal{B} \tau^{* k} \mathcal{K}^{c r i t} A^{\text {crit }}(j) x_{0} \\
& =A^{k} x_{j}^{c r i t}\left(x_{0}\right)+\mathcal{B} \tau^{* k} \bar{\pi}_{+} \tau^{* j} \mathcal{K}^{c r i t} x_{0},
\end{aligned}
$$

where the last equality is by equation (18). The former part in the right of (19) can be decomposed as

$$
\begin{aligned}
A^{k} x_{j}^{c r i t}\left(x_{0}\right) & =A^{k+j} x_{0}+A^{k} \mathcal{B} \tau^{* j} \mathcal{K}^{c r i t} x_{0} \\
= & A^{k+j} x_{0}+\mathcal{B} \tau^{*(k+j)} \pi_{[0, j-1]} \mathcal{K}^{c r i t} x_{0} .
\end{aligned}
$$

The latter part in the right of (19) can be decomposed as

$$
\mathcal{B} \tau^{* k} \bar{\pi}_{+} \tau^{* j} \mathcal{K}^{c r i t} x_{0}=\mathcal{B} \tau^{*(k+j)} \mathcal{K}^{c r i t} x_{0}-\mathcal{B} \tau^{*(k+j)} \pi_{[0, j-1]} \mathcal{K}^{c r i t} x_{0}
$$

Formulae (19), (20) and (21) together show that $A^{\text {crit }}(k) A^{\text {crit }}(j) x_{0}=A^{\text {crit }}(k+j) x_{0}$ for all $x_{0} \in \operatorname{dom}(\mathcal{C})$, thus completing the proof of claim (ii). Also claim (iii) is now quite clear.

Definition 10. The densely defined linear operator $A^{\text {crit }}: H \supset \operatorname{dom}(\mathcal{C}) \rightarrow H$, defined by $A^{\text {crit }}=A^{\text {crit }}(1)$ is called the critical (closed loop) semi-group generator. The family of operators $\left\{\left(A^{\text {crit }}\right)^{j}\right\}_{j \geq 0}$ is called the critical (closed loop) semi-group.

The following lemma describes the common algebraic structure of operators $A^{\text {crit }}, \mathcal{C}^{\text {crit }}$ and $\mathcal{K}^{\text {crit }}$. 
Lemma 11. Let $\Phi=\left[\begin{array}{cc}A^{j} & \mathcal{B}_{\tau^{* j}} \\ \mathcal{C} & \mathcal{D}\end{array}\right]$ be an I/O-stable J-coercive DLS. Then the following equations are valid in $\operatorname{dom}(\mathcal{C})$ :

$$
\begin{aligned}
\mathcal{C}^{c r i t} A^{c r i t} & =\bar{\pi}_{+} \tau^{*} \mathcal{C}^{c r i t} \\
\mathcal{K}^{c r i t} A^{c r i t} & =\bar{\pi}_{+} \tau^{*} \mathcal{K}^{c r i t} .
\end{aligned}
$$

Proof. See the proof of Lemma 9.

Until now we have only given algebraic properties of operators $A^{\text {crit }}, \mathcal{C}^{\text {crit }}$ and $\mathcal{K}^{\text {crit }}$ as possibly unbounded linear mappings on $\operatorname{dom}(\mathcal{C})$. We remark that $\mathcal{C}^{\text {crit }}$ and $\mathcal{K}^{\text {crit }}$ are valid observability maps for a DLS whose semi-group generator is $A^{\text {crit }}$ and state space $\overline{\operatorname{dom}(\mathcal{C})}=H$, provided that certain continuity requirements of these operator are satisfied. In particular, $A^{\text {crit }}$ should be continuous in the norm of $H$. Generally this is not the case.

Basic stability conditions for closed loop semi-group generator $A^{\text {crit }}$ are given in the following lemma. The proof is quite similar to [9, Theorem 50].

Lemma 12. Assume that the $D L S \Phi=\left[\begin{array}{cc}A^{j} & \mathcal{B} \tau^{* j} \\ \mathcal{C} & \mathcal{D}\end{array}\right]$ is I/O-stable and J-coercive. Then the following is true:

(i) $\Phi$ is output stable $\Rightarrow \mathcal{K}^{\text {crit }} \in \mathcal{L}\left(H ; \ell^{2}\left(Z_{+} ; U\right)\right) \Rightarrow K^{\text {crit }}:=\pi_{0} \mathcal{K}^{\text {crit }} \in \mathcal{L}(H ; U) \Rightarrow$ $B K^{\text {crit }} \in \mathcal{L}(U) \Leftrightarrow A^{\text {crit }} \in \mathcal{L}(H)$.

(ii) If $\Phi$ is stable, then $\left\{A^{\text {crit }}(j)\right\}_{j \geq 0} \subset \mathcal{L}(H)$ and there is a constant $C<\infty$ such that

$$
\left\|\left(A^{\text {crit }}\right)^{j}\right\|_{\mathcal{L}(H)} \leq C \quad \forall j \geq 1,
$$

i.e. $A^{\text {crit }}$ is power bounded.

(iii) If $\Phi$ is strongly stable, then

$$
\left(A^{\text {crit }}\right)^{j} x_{0} \rightarrow 0 \quad \forall x_{0} \in H
$$

i.e. $A^{\text {crit }}$ is strongly stable.

Proof. The only not completely trivial part of (i) is the equivalence. This is proved by

$$
A^{\text {crit }}=A+\mathcal{B} \tau^{*} \mathcal{K}^{\text {crit }}=A+\mathcal{B} \pi_{-} \tau^{*} \bar{\pi}_{+} \mathcal{K}^{\text {crit }}=A+B \pi_{0} \mathcal{K}^{\text {crit }},
$$

where range $\left(\pi_{0}\right)$ and $U$ have been identified.

In order to prove claim (ii), we write

$$
\begin{aligned}
& \left\|\left(A^{\text {crit }}\right)^{j}\right\|_{\mathcal{L}(H)}=\left\|A^{j}+\mathcal{B} \tau^{* j} \mathcal{K}^{\text {crit }}\right\|_{\mathcal{L}(H)} \\
& \leq\left\|A^{j}\right\|_{\mathcal{L}(H)}+\|\mathcal{B}\|_{\ell^{2}\left(Z_{+} ; U\right) \rightarrow H}\left\|\mathcal{K}^{c r i t}\right\|_{H \rightarrow \ell^{2}\left(Z_{+} ; U\right)} \leq C<\infty,
\end{aligned}
$$


because $\tau$ is unitary and $A$ is power bounded by assumption. This proves (ii).

The proof of claim (iii) is somewhat similar. Now we estimate for all $x_{0} \in H$

$$
\left\|\left(A^{\text {crit }}\right)^{j} x_{0}\right\|_{H} \leq\left\|A^{j} x_{0}\right\|_{H}+\left\|\mathcal{B} \tau^{* j} \mathcal{K}^{c r i t} x_{0}\right\|_{H} .
$$

Here $A^{j} x_{0} \rightarrow 0$ by the assumed strong stability of $\Phi$. The claim follows once we prove $\mathcal{B} \tau^{* j} \mathcal{K}^{\text {crit }} x_{0} \rightarrow 0$ for all $x_{0} \in H$. Fix $x_{0} \in H$. We have for all $j, J>0$

$$
\begin{aligned}
& \left\|\underline{\mathcal{B}} \tau^{* j} \mathcal{K}^{c r i t} x_{0}\right\|_{H} \\
& <\left\|\underline{\mathcal{B}} \tau^{* j} \pi_{[0, J]} \mathcal{K}^{c r i t} x_{0}\right\|_{H}+\left\|\underline{\mathcal{B}} \tau^{* j} \pi_{[J+1, \infty]} \mathcal{K}^{c r i t} x_{0}\right\|_{H} .
\end{aligned}
$$

The second term on the right of equation (24) gets small by increasing $J$, because $\mathcal{K}^{\text {crit }} x_{0} \in$ $\ell^{2}\left(\mathbf{Z}_{+} ; U\right)$ and $\underline{\mathcal{B}}$ is bounded. Also the first term gets small, as shown by the following inequality, implied by the basic properties of the observability map. For $j>J$

$$
\begin{aligned}
& \left\|\underline{\mathcal{B}} \tau^{* j} \pi_{[0, J]} \tilde{u}\right\|_{H} \leq\left\|A^{j} \underline{\mathcal{B}} \pi_{[0, J]} \tilde{u}\right\|_{H}+\left\|\sum_{i=0}^{j-1} A^{i} B\left(\pi_{[0, J]} \tilde{u}\right)_{j-i-1}\right\|_{H} \\
& =\left\|A^{j-J-1}\left(\sum_{i=0}^{J} A^{i} B u_{J-i}\right)\right\|_{H} \rightarrow 0, \quad \text { for all } \tilde{u} \in \ell^{2}\left(\mathbf{Z}_{+} ; U\right),
\end{aligned}
$$

where the limit follows because $\sum_{i=0}^{J} A^{i} B u_{J-i} \in H$ and $A$ is strongly stable. The proof of the lemma is completed.

The requirement that $K^{\text {crit }} \in \mathcal{L}(H ; U)$ is central in this work. It is sufficient but not necessary to make $A^{\text {crit }}$ bounded. On the other hand, it is necessary for the DLS $\Phi^{e x t}$ of equation (35) to be a DLS, because the input operator of DLS is assumed to be bounded. Two simple sufficient conditions for this conditions are given below:

Proposition 13. Sufficient conditions for $K^{\text {crit }} \in \mathcal{L}(H ; U)$ are

(i) $J \mathcal{C} \in \mathcal{L}\left(H, \ell^{2}\left(\mathbf{Z}_{+} ; Y\right)\right)$,

(ii) the input space $U$ is finite dimensional.

Proof. The first claim is trivial. The second follows because then $K^{\text {crit }}=\pi_{0} \mathcal{K}^{\text {crit }}$ would be a finite dimensional operator.

We end this section by introducing a conjugate symmetric sesquilinear form in $\operatorname{dom}(\mathcal{C}) \times$ $\operatorname{dom}(\mathcal{C}) \subset H \times H$, whose diagonal values give the critical cost. The sesquilinear forms of this kind are basic objects in the Riccati equation system theory of Sections 6 and 7.

Definition 14. Let $J \in \mathcal{L}(Y)$ be self-adjoint and $\Phi=\left[\begin{array}{c}A_{\mathcal{C}}^{j} \\ { }_{\mathcal{C}}{ }^{* j}\end{array}\right]$ be an I/O-stable $J$ coercive DLS. The conjugate symmetric sesquilinear form $P^{\text {crit }}($,$) in \operatorname{dom}(\mathcal{C}) \times \operatorname{dom}(\mathcal{C})$ given by

$$
P^{\text {crit }}\left(x_{0}, x_{1}\right):=\left\langle\mathcal{C}^{c r i t} x_{0}, J \mathcal{C}^{c r i t} x_{1}\right\rangle_{\ell^{2}\left(\mathbf{Z}_{+} ; Y\right)}
$$

is called the critical sesquilinear form associated to $\Phi$ and $J$. 
The I/O-stability of $\Phi$ has an effect to the limit behaviour of $P^{\text {crit }}($, ):

Proposition 15. Let $J \in \mathcal{L}(Y)$ be self-adjoint and $\Phi$ be an I/O-stable and $J$-coercive $D L S$. Then for all $x_{0} \in \operatorname{dom}(\mathcal{C}), \tilde{u} \in \ell^{2}\left(\mathbf{Z}_{+} ; U\right)$

$$
P^{\text {crit }}\left(x_{j}\left(x_{0}, \tilde{u}\right), x_{j}\left(x_{0}, \tilde{u}\right)\right) \rightarrow 0 \quad \text { as } \quad j \rightarrow \infty .
$$

Proof. Fix $\tilde{u} \in \ell^{2}\left(\mathbf{Z}_{+} ; U\right)$ and $x_{0} \in \operatorname{dom}(\mathcal{C})$. We first remark that

$$
\left|P^{c r i t}\left(x_{j}\left(x_{0}, \tilde{u}\right), x_{j}\left(x_{0}, \tilde{u}\right)\right)\right| \leq\|J\| \cdot\left\|\mathcal{C}^{c r i t} x_{j}\left(x_{0}, \tilde{u}\right)\right\|^{2} \leq\|J\| \cdot\|\Pi\|^{2} \cdot\left\|\mathcal{C} x_{j}\left(x_{0}, \tilde{u}\right)\right\|^{2},
$$

where $\Pi$ is the bounded projection introduced just after Definition 7 . So it suffices to show that $\mathcal{C} x_{j}\left(x_{0}, \tilde{u}\right) \rightarrow 0$. We have

$$
\mathcal{C} x_{j}\left(x_{0}, \tilde{u}\right)=\bar{\pi}_{+} \tau^{* j} \mathcal{C} x_{0}+\mathcal{C B} \tau^{* j} \bar{\pi}_{+} \tilde{u}=\bar{\pi}_{+} \tau^{* j} \mathcal{C} x_{0}+\bar{\pi}_{+} \mathcal{D} \pi_{-} \tau^{* j} \bar{\pi}_{+} \tilde{u} .
$$

The first part of equation (25) approaches zero, because $\mathcal{C} x_{0} \in \ell^{2}\left(\mathbf{Z}_{+} ; Y\right)$. For the second part, write

$$
\left(\bar{\pi}_{+} \mathcal{D} \pi_{-} \tau^{* j}\right) \bar{\pi}_{+} \tilde{u}=\left(\bar{\pi}_{+} \mathcal{D} \pi_{-} \tau^{* j}\right) \pi_{[0, J]} \tilde{u}+\left(\bar{\pi}_{+} \mathcal{D} \pi_{-} \tau^{* j}\right) \pi_{[J+1, \infty]} \tilde{u} .
$$

Let $\epsilon>0$ be arbitrary. Choose $J$ so large that $\left\|\pi_{[J+1, \infty]} \tilde{u}\right\|_{\ell^{2}\left(\mathbf{Z}_{+} ; U\right)}<\epsilon /\left(2\|\mathcal{D}\| \|_{\ell^{2}\left(\mathbf{Z}_{+} ; U\right) \rightarrow \ell^{2}\left(\mathbf{Z}_{+} ; Y\right)}\right.$ which gives immediately $\left\|\left(\bar{\pi}_{+} \mathcal{D} \pi_{-} \tau^{* j}\right) \pi_{[J+1, \infty]} \tilde{u}\right\|<\epsilon / 2$. For $j>J$ write

$$
\bar{\pi}_{+} \mathcal{D} \pi_{-} \tau^{* j} \pi_{[0, J]} \tilde{u}=\left(\bar{\pi}_{+} \tau^{* j}\right) \mathcal{D} \pi_{[0, J]} \tilde{u} .
$$

By I/O-stability, $\mathcal{D} \pi_{[0, J]} \in \ell^{2}\left(\mathbf{Z}_{+} ; Y\right)$ and the above expression can be made less that $\epsilon / 2$ by increasing $j$. So the second term in (25) approaches zero as $j$ increases. This completes the proof.

In the following proposition, the last one of this section, we separate the cost of input into two parts, the first of which does not depend on the control $\tilde{u}$ we are applying, but only on the initial value $x_{0}$. The second part of the cost depends only on the deviation from the criticality of the applied input $\tilde{u}$.

Proposition 16. Let $J \in \mathcal{L}(Y)$ be self-adjoint and $\Phi=\left[\begin{array}{c}A_{\mathcal{C}}^{j} \\ \mathcal{B}_{\mathcal{D}}^{* j}\end{array}\right]$ be an I/O-stable $J$ coercive DLS. Then the cost functional can be separated in the following way:

$$
J\left(x_{0}, \tilde{u}\right)=J\left(x_{0}, \tilde{u}^{\text {crit }}\left(x_{0}\right)\right)+J\left(0, \tilde{u}-\tilde{u}^{c r i t}\left(x_{0}\right)\right)
$$

for all input functions $\tilde{w} \in \ell^{2}(\mathbf{Z} ; U)$. Moreover, we have

$$
P^{\text {crit }}\left(x_{0}, x_{0}\right)=J\left(x_{0}, \tilde{u}^{\text {crit }}\left(x_{0}\right)\right),
$$

where $P($,$) is defined in Definition 14$.

Proof. Define $\tilde{w}:=\tilde{u}-\tilde{u}^{\text {crit }}\left(x_{0}\right) \in \ell^{2}\left(\mathbf{Z}_{+} ; Y\right)$. Then quite easily

$$
\begin{aligned}
& J\left(x_{0}, \tilde{u}\right)=J\left(x_{0}, \tilde{u}^{\text {crit }}\left(x_{0}\right)+\tilde{w}\right) \\
& =J\left(x_{0}, u^{c r i t}\left(x_{0}\right)\right)+2 \operatorname{Re}\left\langle\bar{\pi}_{+} \mathcal{D}^{*} J \mathcal{C} x_{0}+\bar{\pi}_{+} \mathcal{D}^{*} J \mathcal{D} \tilde{u}^{c r i t}\left(x_{0}\right), \tilde{w}\right\rangle_{\ell^{2}\left(\mathbf{Z}_{+} ; Y\right)}+J(0, w)
\end{aligned}
$$

But now the middle term in the left of (28) vanishes, because the critical cost satisfies formula (9). This immediately proves (26). Equation (27) is immediate from the definition of $\mathcal{C}^{\text {crit }}$. 


\section{Factorization of the I/O-map and the Popov operator}

In this section we consider certain factorizations of I/O-map of an I/O-stable DLS. The approach is similar to that given in [20], [22]. The following definitions give us the basic tools needed in the factorization of the Popov operator $\bar{\pi}_{+} \mathcal{D}^{*} J \mathcal{D} \bar{\pi}_{+}$. We note that the operator $J$ of this section will ultimately appear be the same $J$ as in formula (8) defining the cost functional. We shall frequently use the notion of "bounded causal shift invariant operator". This can always be regarded as an I/O-map of an I/O-stable DLS (see [9, Lemma 8]).

Definition 17. Let $J \in \mathcal{L}(Y)$ be self-adjoint, and let $S \in \mathcal{L}(U)$ self-adjoint and invertible. Let $\mathcal{D}$ be the I/O-map of an I/O-stable DLS.

(i) The operator $E \in \mathcal{L}(U)$ is $S$-unitary, if it is boundedly invertible and $E^{*} S E=S$.

(ii) The causal shift invariant operator $\mathcal{N} \in \mathcal{L}\left(\ell^{2}(\mathbf{Z} ; U), \ell^{2}(\mathbf{Z} ; Y)\right)$ is $(J, S)$-inner, if $\mathcal{N}^{*} J \mathcal{N}=S$.

(iii) The causal shift invariant operator $\mathcal{X} \in \mathcal{L}\left(\ell^{2}(\mathbf{Z} ; U)\right)$ is outer, if $\overline{\text { range }\left(\mathcal{X}_{+}\right)}=$ $\ell^{2}\left(\mathbf{Z}_{+} ; U\right)$.

(iv) The causal shift invariant operator $\mathcal{X} \in \mathcal{L}\left(\ell^{2}\left(\mathbf{Z}_{+} ; U\right)\right)$ is $S$-spectral factor of $\mathcal{D}^{*} J \mathcal{D}$, if $\mathcal{X}$ has a bounded causal shift invariant inverse $\mathcal{X}^{-1}$ in $\ell^{2}(\mathbf{Z} ; U)$ and $\mathcal{D}^{*} J \mathcal{D}=\mathcal{X}^{*} S \mathcal{X}$.

The following special factorization of an I/O-stable I/O-map is necessary:

Definition 18. Let $J \in \mathcal{L}(Y)$ be self-adjoint, and let $S \in \mathcal{L}(U)$ be self-adjoint and invertible. Let $\mathcal{D}$ be the I/O-map of an I/O-stable DLS. Then the pair of operators $(\mathcal{N}, \mathcal{X})$ is an $(J, S)$-inner-outer factorization of $\mathcal{D}$, if the following conditions hold:

(i) $\mathcal{N} \in \mathcal{L}\left(\ell^{2}(\mathbf{Z} ; U), \ell^{2}(\mathbf{Z} ; Y)\right)$ and $\mathcal{X} \in \mathcal{L}\left(\ell^{2}(\mathbf{Z} ; U)\right)$ are causal shift invariant operators,

(ii) $\mathcal{N}$ is $(J, S)$-inner,

(iii) $\mathcal{X}$ is outer,

(iv) $\mathcal{D}=\mathcal{N} \mathcal{X}$

If, in addition $\mathcal{X}$ is injective and range $\left(\mathcal{X} \bar{\pi}_{+}\right)=\ell^{2}\left(\mathbf{Z}_{+} ; U\right)$, we say that the outer part $\mathcal{X}$ of the factorization $(\mathcal{N}, \mathcal{X})$ has a bounded inverse.

The latter is equivalent with saying that the outer Toeplitz operator $\mathcal{X} \bar{\pi}_{+}$is coercive and has a bounded inverse.

We start with proving a simple and frequently used proposition: 
Proposition 19. Let $\mathcal{D}$ be the I/O-map of an I/O-stable DLS. Let $(\mathcal{N}, \mathcal{X})$ is an $(J, S)$ inner-outer factorization of $\mathcal{D}$, such that the outer part $\mathcal{X}$ of the factorization has a bounded inverse. Define the static part of the outer factor by $X=\pi_{0} \mathcal{X} \pi_{0} \in \mathcal{L}(U)$, with the identification of spaces range $\left(\pi_{0}\right)$ and $U$. Then $X^{-1} \in \mathcal{L}(U)$ and $X^{-1}=\pi_{0} \mathcal{X}^{-1} \pi_{0}$.

Proof. We can write by the causality $\pi_{0}=\pi_{0}\left(\mathcal{X} \bar{\pi}_{+}\right)^{-1}\left(\mathcal{X} \bar{\pi}_{+}\right) \pi_{0}=\pi_{0}\left(\mathcal{X} \bar{\pi}_{+}\right)^{-1} \pi_{0} \cdot \pi_{0} \mathcal{X} \pi_{0}$ and similarly $\pi_{0}=\pi_{0} \mathcal{X} \pi_{0} \cdot \pi_{0}\left(\mathcal{X} \bar{\pi}_{+}\right)^{-1} \pi_{0}$. Identifying $\pi_{0}$ with the identity operator in $\mathcal{L}(U)$, we see that $X$ is a bounded bijection on $U$. It thus has a bounded inverse as claimed.

$S$ is called the sensitivity operator of the factorization in [24]. There is a strong link between $S$-spectral factorizations of $\mathcal{D}^{*} J \mathcal{D}$ and $(J, S)$-inner-outer factorizations of $\mathcal{D}$ :

Proposition 20. Let $\mathcal{D}$ be the I/O-map of an I/O-stable DLS. Then the following are equivalent:

(i) $(\mathcal{N}, \mathcal{X})$ is an $(J, S)$-inner-outer factorization of $\mathcal{D}$, with the outer part $\mathcal{X}$ having a bounded inverse,

(ii) $\mathcal{X}$ is a spectral factor of $\mathcal{D}^{*} J \mathcal{D}$, and $\mathcal{N}=\mathcal{D} \mathcal{X}^{-1}$.

Proof. Let us first show that (i) implies (ii). Assume that $(\mathcal{N}, \mathcal{X})$ is a $(J, S)$-inner-outer factorization of $\mathcal{D}=\mathcal{N} \mathcal{X}^{-1}$. Then

$$
\mathcal{D}^{*} J \mathcal{D}=\mathcal{X}^{*}\left(\mathcal{N}^{*} J \mathcal{N}\right)(\mathcal{X})=\mathcal{X}^{*} S \mathcal{X}
$$

Because $\mathcal{X}^{-1}$ is causal and shift invariant, $\mathcal{X}$ is a $S$-spectral factor if $\mathcal{X}^{-1}$ is bounded. We conclude this the fact that the Toeplitz operator $\mathcal{X} \bar{\pi}_{+}$has a bounded inverse.

By the causality of both $\mathcal{X}$ and $\mathcal{X}^{-1},\left(\mathcal{X} \bar{\pi}_{+}\right)^{-1}=\mathcal{X}^{-1} \bar{\pi}_{+}$, which is now bounded. We can extend $\mathcal{X}^{-1} \bar{\pi}_{+}$uniquely to $\ell^{2}(\mathbf{Z} ; U) \cap S e q(U)$ by the shift invariance, and then uniquely to $\ell^{2}(\mathbf{Z} ; U)=\overline{\ell^{2}(\mathbf{Z} ; U) \cap S e q(U)}$ by the continuity. This bounded extension coincides with $\mathcal{X}^{-1}$ in the range of $\mathcal{X}$, proving that $\mathcal{X}^{-1}$ is bounded. The first part of the proposition now follows.

To show that (ii) implies (i), assume that we have the spectral factorization $\mathcal{D}^{*} J \mathcal{D}=$ $\mathcal{X}^{*} S \mathcal{X}$. Define $\mathcal{N}:=\mathcal{D} \mathcal{X}^{-1}$. Then $\mathcal{N}$ is a bounded causal and shift invariant operator, satisfying $\mathcal{D}=\mathcal{N} \mathcal{X}$. The factor $\mathcal{N}$ satisfies

$$
\mathcal{N}^{*} J \mathcal{N}=\left(\mathcal{X}^{-1}\right)^{*}\left(\mathcal{D}^{*} J \mathcal{D}\right)\left(\mathcal{X}^{-1}\right)=\left(\mathcal{X}^{-1}\right)^{*}\left(\mathcal{X}^{*} S \mathcal{X}\right)\left(\mathcal{X}^{-1}\right)=S
$$

which proves that $\mathcal{N}$ is $(J, S)$-inner. It follows that $(\mathcal{N}, \mathcal{X})$ is a $(J, S)$-inner-outer factorization of $\mathcal{D}$, with $\mathcal{X}$ having a bounded inverse. The remaining part of the proposition is thus proved. 
Not all operators of form $\mathcal{D}^{*} J \mathcal{D}$ have $S$-spectral factorization for any $S$. Those that have the factorization are more interesting to us. If we know one $(J, S)$-inner-outer factorization of $\mathcal{D}$ for some $S$, then we know them all. This is because all the $(J, S)$-inner-outer factorization can be parameterized by the set of all $S$-unitary operators.

Proposition 21. Let $J \in \mathcal{L}(Y)$ be self-adjoint and $\mathcal{D}$ be the I/O-map of an I/O-stable $D L S$. Let $(\mathcal{N}, \mathcal{X})$ be a $(J, S)$-inner-outer factorization of $\mathcal{D}$ for some $S \in \mathcal{L}(U)$ with $\mathcal{X}$ having a bounded inverse. Then the set of all possible $\left(J, S_{E}\right)$-inner-outer factorizations $\left(\mathcal{N}_{E}, \mathcal{X}_{E}\right)$ of $\mathcal{D}$ can be parameterized by

$$
\mathcal{N}_{E}=\mathcal{N} E, \quad \mathcal{X}_{E}=E^{-1} \mathcal{X}, \quad S_{E}=E^{*} S E,
$$

where $E$ ranges over the set all boundedly invertible operators in $\mathcal{L}(U)$. In particular, if we in addition require that $S_{E}=S$, the $E$ is allowed to range over the set of all S-unitary operators $E \in \mathcal{L}(U)$.

Proof. We first show that for each invertible $E$ we have the factorization as claimed. So let $E \in \mathcal{L}(U)$ be boundedly invertible and $(\mathcal{N}, \mathcal{X})$ be a $(J, S)$-inner-outer factorization of $\mathcal{D}$ for some $S \in \mathcal{L}(U)$. Trivially $\mathcal{D}=\mathcal{N} \mathcal{X}=\mathcal{N}_{E} \mathcal{X}_{E}$. Also $\mathcal{N}_{E}, \mathcal{X}_{E}$ and $\mathcal{X}_{E}^{-1}$ are bounded causal shift invariant operators. Because

$$
\mathcal{N}_{E}^{*} J \mathcal{N}_{E}=(\mathcal{N} E)^{*} J(\mathcal{N} E)=E^{*} \mathcal{N}^{*} J \mathcal{N} E=E^{*} S E=: S_{E},
$$

$\mathcal{N}_{E}$ is $\left(J, S_{E}\right)$-inner.

In order to prove the remaining part, we must show that if there is another $\left(J, S^{\prime}\right)$-innerouter factorization $\left(\mathcal{N}^{\prime}, \mathcal{X}^{\prime}\right)$, then it is of form $\left(\mathcal{N}_{E}, \mathcal{X}_{E}\right)$ for some boundedly invertible $E \in \mathcal{L}(U)$. Both $\left(\mathcal{N}^{\prime}, \mathcal{X}^{\prime}\right),(\mathcal{N}, \mathcal{X})$ satisfy

$$
\mathcal{D}=\mathcal{N} \mathcal{X}=\mathcal{N}^{\prime} \mathcal{X}^{\prime}
$$

Because both $\mathcal{X}$ and $\mathcal{X}^{\prime}$ together with their inverses are bounded, causal and shift invariant, both the operators $\mathcal{U}:=\mathcal{X}^{\prime} \mathcal{X}^{-1}$ and $\mathcal{U}^{-1}:=\mathcal{X}\left(\mathcal{X}^{\prime}\right)^{-1}$ are bounded causal shift invariant operators. We have then

$$
\mathcal{N}=\mathcal{N}^{\prime} \mathcal{U}
$$

Now, because $\mathcal{N}$ is $(J, S)$-inner and $\mathcal{N}^{\prime}$ is $\left(J, S^{\prime}\right)$-inner

$$
S=\mathcal{N}^{*} J \mathcal{N}=\left(\mathcal{N}^{\prime} \mathcal{U}\right)^{*} J\left(\mathcal{N}^{\prime} \mathcal{U}\right)=\mathcal{U}^{*}\left(\mathcal{N}^{\prime *} J \mathcal{N}^{\prime}\right) \mathcal{U}=\mathcal{U}^{*} S^{\prime} \mathcal{U}
$$

which implies immediately

$$
S \mathcal{U}^{-1}=\mathcal{U}^{*} S^{\prime}
$$

Both $S$ and $S^{\prime}$ are static operators. $\mathcal{U}^{*}$ is anti-causal and $\mathcal{U}^{-1}$ causal. The the right side of equation (31) is causal and the left side is anti-causal. So the both sides of equation (31) are static, and thus $\mathcal{U}^{-1}$ must be equal to a multiplication by some $E \in \mathcal{L}(U)$ with bounded inverse. This together with equation (30) implies $\mathcal{N}^{\prime}=\mathcal{N} E=\mathcal{N}_{E}$ and also by the definition of $\mathcal{U}$ we obtain $\mathcal{X}^{\prime}=E^{-1} \mathcal{X}=\mathcal{X}_{E}$. Finally (31) gives $S^{\prime}=E^{*} S E=S_{E}$. The statement about the $S$-unitary parameterizations is trivial, and the proof of the proposition is now completed. 
The existence of $(J, S)$-inner-outer factorization of $\mathcal{D}$ will provide us with useful information about the properties of the the Toeplitz operator $\bar{\pi}_{+} \mathcal{D}^{*} J \mathcal{D} \bar{\pi}_{+}$. The following lemma is the main result of this section:

Lemma 22. Let $\Phi=\left[\begin{array}{cc}A_{\mathcal{C}}^{j} & \mathcal{B}_{\mathcal{D}}^{* j} \\ \mathcal{D}\end{array}\right]$ be an I/O-stable DLS. Let $J \in \mathcal{L}(Y)$ be self-adjoint and $S \in \mathcal{L}(U)$ self adjoint with bounded inverse. If $\mathcal{D}$ has a $(J, S)$-inner-outer factorization $(\mathcal{N}, \mathcal{X})$ with $\mathcal{X}$ having a bounded inverse, then the following holds:

(i) $\Phi$ is J-coercive.

(ii) The inverse of the Popov operator operator $\bar{\pi}_{+} \mathcal{D}^{*} J \mathcal{D} \bar{\pi}_{+}$satisfies

$$
\left(\bar{\pi}_{+} \mathcal{D}^{*} J \mathcal{D} \bar{\pi}_{+}\right)^{-1}=\left(\bar{\pi}_{+} \mathcal{X}^{-1} \bar{\pi}_{+}\right) S^{-1}\left(\bar{\pi}_{+}\left(\mathcal{X}^{*}\right)^{-1} \bar{\pi}_{+}\right)
$$

(iii) The critical operators $A^{\text {crit }}, \mathcal{C}^{\text {crit }}$ and $\mathcal{K}^{\text {crit }}$ can be written in forms

$$
\begin{aligned}
& A^{\text {crit }}=A-\mathcal{B} \mathcal{X}^{-1} \tau^{*} S^{-1} \bar{\pi}_{+} \mathcal{N}^{*} J \mathcal{C}, \\
& \mathcal{C}^{\text {crit }}=\mathcal{C}-\mathcal{N} S^{-1} \bar{\pi}_{+} \mathcal{N}^{*} J \mathcal{C} \\
& \mathcal{K}^{c r i t}=-\mathcal{X}^{-1} S^{-1} \bar{\pi}_{+} \mathcal{N}^{*} J \mathcal{C}
\end{aligned}
$$

Proof. We prove parts (i) and (ii) at the same time. Given $\bar{\pi}_{+} \tilde{f} \in \ell^{2}\left(\mathbf{Z}_{+} ; U\right)$, we try to solve the equation

$$
\bar{\pi}_{+} \mathcal{D}^{*} J \mathcal{D} \bar{\pi}_{+} \tilde{u}=\bar{\pi}_{+} \tilde{f}
$$

for $\bar{\pi}_{+} \tilde{u}$. Replace $\mathcal{D}$ by $\mathcal{N} \mathcal{X}$ and use the fact that $\mathcal{N}$ is $(J, S)$-inner to get

$$
\bar{\pi}_{+} \tilde{f}=\bar{\pi}_{+} \mathcal{X}^{*} S \mathcal{X} \bar{\pi}_{+} \tilde{u}
$$

Applying $S^{-1} \bar{\pi}_{+}\left(\mathcal{X}^{*}\right)^{-1}$ to this equation, and using the anti-causality of $\mathcal{X}^{*}$ and causality of $M^{-1}$ gives

$$
\begin{aligned}
& \left(S^{-1} \bar{\pi}_{+}\left(\mathcal{X}^{*}\right)^{-1}\right) \bar{\pi}_{+} \tilde{f}=\left(S^{-1} \bar{\pi}_{+}\left(\mathcal{X}^{*}\right)^{-1}\right)\left(\bar{\pi}_{+} \mathcal{X}^{*} S \mathcal{X}_{+}\right) \tilde{u} \\
& =S^{-1}\left(\bar{\pi}_{+}\left(\mathcal{X}^{*}\right)^{-1} \bar{\pi}_{+} \cdot \bar{\pi}_{+} \mathcal{X}^{*} \bar{\pi}_{+}\right) S \mathcal{X} \bar{\pi}_{+} \tilde{u}=S^{-1}\left(\bar{\pi}_{+}\left(\mathcal{X}^{*}\right)^{-1} \mathcal{X}^{*} \bar{\pi}_{+}\right) S \mathcal{X} \bar{\pi}_{+} \tilde{u}=\mathcal{X} \bar{\pi}_{+} \tilde{u}
\end{aligned}
$$

which is equivalent to

$$
\bar{\pi}_{+} \tilde{u}=\mathcal{X}^{-1} S^{-1} \bar{\pi}_{+}\left(\mathcal{X}^{*}\right)^{-1} \bar{\pi}_{+} \tilde{f} .
$$

This $\bar{\pi}_{+} \tilde{u}$ is the only possible solution to equation (32), and accordingly $\bar{\pi}_{+} \mathcal{D}^{*} J \mathcal{D} \bar{\pi}_{+}$is injective in $\ell^{2}\left(Z_{+} ; U\right)$.

To check that this really is a solution, it suffices to compute

$$
\begin{aligned}
& \left(\bar{\pi}_{+} \mathcal{D}^{*} J \mathcal{D} \bar{\pi}_{+}\right) \mathcal{X}^{-1} S^{-1} \bar{\pi}_{+}\left(\mathcal{X}^{*}\right)^{-1} \bar{\pi}_{+} \tilde{f}=\bar{\pi}_{+} \mathcal{D}^{*} J\left(\mathcal{D} \mathcal{X}^{-1}\right) S^{-1} \bar{\pi}_{+}\left(\mathcal{X}^{*}\right)^{-1} \bar{\pi}_{+} \tilde{f} \\
& =\bar{\pi}_{+} \mathcal{X}^{*}\left(\mathcal{N} J \mathcal{N} S^{-1}\right) \bar{\pi}_{+}\left(\mathcal{X}^{*}\right)^{-1} \bar{\pi}_{+} \tilde{f}=\left(\bar{\pi}_{+} \mathcal{X}^{*} S\left(\mathcal{X}^{*}\right)^{-1} \bar{\pi}_{+}\right) \bar{\pi}_{+} \tilde{f}=\bar{\pi}_{+} \tilde{f}
\end{aligned}
$$

So there is a solution for each $\bar{\pi}_{+} \tilde{f} \in \ell^{2}\left(\mathbf{Z}_{+} ; U\right)$, and it follows that $\bar{\pi}_{+} \mathcal{D}^{*} J \mathcal{D} \bar{\pi}_{+}$is surjective. 
Thus $\bar{\pi}_{+} \mathcal{D}^{*} J \mathcal{D} \bar{\pi}_{+}$is a bounded bijection between two Hilbert spaces. It follows that $\bar{\pi}_{+} \mathcal{D}^{*} J \mathcal{D} \bar{\pi}_{+}$must have a bounded inverse; i.e. $\Phi$ is $J$-coercive. The inverse is given by formula (34). This proves the first two claims of the lemma. In order to prove the remaining claim (iii), is is sufficient to apply the formula of claim (ii) to the formulae of Definition 7 . This completes the proof of the lemma.

Corollary 23. Assume that $\mathcal{D}$ is an I/O-map of an I/O-stable DLS $\Phi$ having a $(J, S)$ inner-outer factorization $(\mathcal{N}, \mathcal{X})$. Then $\mathcal{X} \bar{\pi}_{+}$has a bounded inverse if and only if $\Phi$ is $J$-coercive. When the equivalence holds, then $S^{-1} \in \mathcal{L}(U)$.

Proof. The "if" part is proved as follows. For a $(J, S)$-inner-outer factorization $(\mathcal{N}, \mathcal{X})$ we have $\bar{\pi}_{+} \mathcal{D}^{*} J \mathcal{D} \bar{\pi}_{+}=\bar{\pi}_{+} \mathcal{X}^{*} S \mathcal{X} \bar{\pi}_{+}$. The bounded, causal and shift invariant operator $\mathcal{X}$ is an I/O-map of an I/O-stable DLS. From $J$-coercivity of $\Phi$ it follows further that this DLS is $S$-coercive, too. Now range $\left(\mathcal{X} \bar{\pi}_{+}\right)$is closed, by Proposition 6 . The "only if" part is claim (i) of Lemma 22. The remaining claim follows by writing $S=\left(\left(\mathcal{X} \bar{\pi}_{+}\right)^{*}\right)^{-1}\left(\bar{\pi}_{+} \mathcal{D}^{*} J \mathcal{D} \bar{\pi}_{+}\right)\left(\mathcal{X} \bar{\pi}_{+}^{*}\right)^{-1}$. So the (static) operator $S$ has a bounded inverse in $\mathcal{L}\left(\ell^{2}\left(\mathbf{Z}_{+} ; U\right)\right)$ and immediately also in $\mathcal{L}(U)$ (see [22, Lemma 14]).

\section{The critical control in feedback form}

In this section we give necessary and sufficient conditions for a class of critical control problems to be of the feedback form as defined below. This class is associated to I/Ostable and $J$-coercive DLS's, with the additional requirement that the critical one step feedback operator $K^{\text {crit }}=\pi_{0} \mathcal{K}^{\text {crit }}$ is bounded. We remark that this latter requirement is imposed on the common structure of $\Phi$ and $J$, and not on these objects separately. The exact formulations and proofs of the results are divided into two Lemmas 25 and 26, and then stated in Theorems 27 and 28.

Let $\Phi=\left[\begin{array}{cc}A^{j} & \mathcal{B} \tau^{* j} \\ \mathcal{C} & \mathcal{D}\end{array}\right]$ be an I/O-stable and $J$-coercive DLS, with $K^{\text {crit }}$ bounded. We have seen in Lemma 11 that the closed loop feedback map $\mathcal{K}^{\text {crit }}$ is a valid observability map for a DLS having the critical semi-group generator $A^{\text {crit }}$ as its semi-group generator, provided that no trouble emerges with the right hand column of the DLS in question. This gives us a reason to ask the following question: Is there an I/O-stable feedback pair $[\mathcal{K}, \mathcal{F}]$ for the original DLS $\Phi$ such that the extended system

$$
\Phi^{e x t}:=[\Phi,[\mathcal{K}, \mathcal{F}]]=\left(\begin{array}{cc}
A^{j} & \mathcal{B} \tau^{* j} \\
{\left[\begin{array}{c}
\mathcal{C} \\
\mathcal{K}
\end{array}\right]} & {\left[\begin{array}{c}
\mathcal{D} \\
\mathcal{F}
\end{array}\right]}
\end{array}\right)
$$

has the following properties: 
(i) Both the extended DLS $\Phi^{e x t}$ and the closed loop extended DLS

$$
\begin{aligned}
& \Phi_{\diamond}^{\text {ext }}:=[\Phi,[\mathcal{K}, \mathcal{F}]]_{\diamond}:=\left[\begin{array}{cc}
A_{\diamond}^{j} & \mathcal{B}_{\diamond} \tau^{* j} \\
{\left[\begin{array}{c}
\mathcal{C}_{\diamond} \\
\mathcal{K}_{\diamond}
\end{array}\right]} & {\left[\begin{array}{c}
\mathcal{D}_{\diamond} \\
\mathcal{F}_{\diamond}
\end{array}\right]}
\end{array}\right] \\
& =\left[\begin{array}{cl}
A^{j}+\mathcal{B} \tau^{* j}(I-\mathcal{F})^{-1} \mathcal{K} & \mathcal{B} \tau^{* j}(I-\mathcal{F})^{-1} \\
{\left[\begin{array}{c}
\mathcal{C}+\mathcal{D}(I-\mathcal{F})^{-1} \mathcal{K} \\
(\mathcal{I}-\mathcal{F})^{-1} \mathcal{K}
\end{array}\right]} & {\left[\begin{array}{c}
\mathcal{D}(\mathcal{I}-\mathcal{F})^{-1} \\
(\mathcal{I}-\mathcal{F})^{-1}-I
\end{array}\right]}
\end{array}\right]
\end{aligned}
$$

are I/O-stable.

(ii) With initial value $x_{0} \in \operatorname{dom}(\mathcal{C})$ and zero input, $\Phi_{\diamond}^{e x t}$ outputs the critical state sequence $\left\{x_{j}^{\text {crit }}\left(x_{0}\right)\right\}_{j \geq 0}$, critical output $\tilde{y}^{\text {crit }}\left(x_{0}\right)$ and critical control $\tilde{u}^{\text {crit }}\left(x_{0}\right)$ of the original system $\Phi$.

For a fairly thorough exposition of the feedback DLS's and their stability properties, see [9]. The feedback connection and signals of the closed loop system $\Phi_{\diamond}^{e x t}$ are illustrated in the following figure:

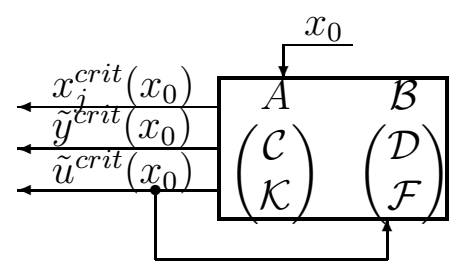

We give a name for this situation:

Definition 24. Let $J \in \mathcal{L}(Y)$ be self-adjoint and $\Phi=\left[\begin{array}{c}A_{\mathcal{C}}^{j} \mathcal{B}_{\mathcal{D}}^{\tau^{* j}}\end{array}\right]$ be an I/O-stable $J$ coercive DLS. If its critical states, outputs and controls are of the form described above, we say that the critical control of $\Phi$ is the of feedback form. The feedback pair $[\mathcal{K}, \mathcal{F}]$ is called a critical feedback for $\Phi$. 
Now we have made enough preparations to attack the first part of our problem.

Lemma 25. Let $\Phi=\left[\begin{array}{cc}A_{\mathcal{C}}^{j} & \mathcal{B} \tau^{* j} \\ \mathcal{D}\end{array}\right]$ be an I/O-stable DLS and $J \in \mathcal{L}(Y)$ self-adjoint. Assume that the following holds:

(i) $K^{\text {crit }}=\pi_{0} \mathcal{K}^{\text {crit }}$ is bounded,

(ii) $\mathcal{D}$ has a $(J, S)$-inner-outer factorization $(\mathcal{N}, \mathcal{X})$ such the outer part $\mathcal{X}$ has a bounded inverse.

Then the following holds:

(i) $\Phi$ is $J$-coercive.

(ii) $[\mathcal{K}, \mathcal{F}]$ is an $I / O$-stable feedback pair for $\Phi$, where the operators $\mathcal{K}, \mathcal{F}$ are defined by

$$
\begin{aligned}
\mathcal{K} & :=-S^{-1} \bar{\pi}_{+} \mathcal{N}^{*} J \mathcal{C}, \\
\mathcal{F} & :=\mathcal{I}-\mathcal{X}
\end{aligned}
$$

(iii) The critical control of $\Phi$ is of the feedback form with the critical feedback pair $[\mathcal{K}, \mathcal{F}]$.

(iv) If, in addition, $\Phi$ is output stable, then $[\mathcal{K}, \mathcal{F}]$ is a stable feedback pair for $\Phi ;$ i.e. $\mathcal{K}$ is bounded.

Proof. Claim (i) follows directly from Corollary 23. In order to prove claim (ii) we show that $\left[\begin{array}{cc}A^{j} & \mathcal{B} \tau^{* j} \\ \mathcal{K} & \mathcal{F}\end{array}\right]$ is an I/O-stable DLS. We have

$$
\begin{aligned}
& \mathcal{K} A=-S^{-1} \bar{\pi}_{+} \mathcal{N}^{*} J \mathcal{C} A=-S^{-1} \bar{\pi}_{+} \mathcal{N}^{*} \bar{\pi}_{+} \tau^{*} J \mathcal{C}=-S^{-1} \bar{\pi}_{+} \mathcal{N}^{*} \tau^{*} J \mathcal{C} \\
& =-S^{-1} \bar{\pi}_{+} \tau^{*} \mathcal{N}^{*} J \mathcal{C}=-\bar{\pi}_{+} \tau^{*}\left(S^{-1} \bar{\pi}_{+} \mathcal{N}^{*} J \mathcal{C}\right)=\bar{\pi}_{+} \tau^{*} \mathcal{K},
\end{aligned}
$$

where we used the fact that $\mathcal{N}^{*}$ is anti-causal and shift invariant, and $S$ is a static operator. Furthermore, a similar calculation yields

$$
\begin{aligned}
\mathcal{K B} & =-S^{-1} \bar{\pi}_{+} \mathcal{N}^{*} J \mathcal{C B}=-S^{-1} \bar{\pi}_{+} \mathcal{N}^{*}\left(\bar{\pi}_{+} J \mathcal{D} \pi_{-}\right) \\
& =-S^{-1} \bar{\pi}_{+} \mathcal{N}^{*} J \mathcal{D} \pi_{-}=-S^{-1} \bar{\pi}_{+}\left(\mathcal{N}^{*} J \mathcal{N}\right) \mathcal{X} \pi_{-} \\
& =-\bar{\pi}_{+} S^{-1} S \mathcal{X} \pi_{-}=-\bar{\pi}_{+} \mathcal{X}_{\pi_{-}}=-\bar{\pi}_{+}(\mathcal{I}-\mathcal{F}) \pi_{-}=\bar{\pi}_{+} \mathcal{F} \pi_{-} .
\end{aligned}
$$

So the pair $[\mathcal{K}, \mathcal{F}]$ interacts in the expected way with $A$ and $\mathcal{B}$.

By Proposition 19, $\pi_{0}(\mathcal{I}-\mathcal{F})^{-1} \pi_{0}=\pi_{0} \mathcal{X}^{-1} \pi_{0}=X^{-1}$, where $X, X^{-1} \in \mathcal{L}(U)$. Now we obtain

$$
K^{\text {crit }}=\pi_{0} \mathcal{K}^{\text {crit }}=\pi_{0}(\mathcal{I}-\mathcal{F})^{-1} \mathcal{K}=\pi_{0}(\mathcal{I}-\mathcal{F})^{-1} \pi_{0} \mathcal{K}=X^{-1} \pi_{0} \mathcal{K} .
$$

Because $\pi_{0} \mathcal{K}^{\text {crit }}$ is bounded by assumption, so is $\pi_{0} \mathcal{K}$. Now we have proved that $\left[\begin{array}{c}A^{j} \mathcal{B} \tau_{\mathcal{F}}^{* j} \\ \mathcal{F}^{2}\end{array}\right]$ is an I/O-stable DLS, if we just note that $\mathcal{F}=\mathcal{I}-\mathcal{X}$ is a bounded operator, because $\mathcal{X}$ is. 
It follows directly from equation (37) that $\operatorname{dom}(\mathcal{C}) \subset\left\{x_{0} \in H \mid \mathcal{K} x_{0} \in \ell^{2}\left(\mathbf{Z}_{+} ; U\right)\right\}=$ $\operatorname{dom}(\mathcal{K})$ because all the other operators $S^{-1}, \mathcal{N}^{*}$ and $J$ are bounded operators between appropriate spaces. Finally we see that $\mathcal{I}-\mathcal{F}=\mathcal{X}$ has a bounded causal and shift invariant inverse. So the pair $[\mathcal{K}, \mathcal{F}]$ is an I/O-stable feedback pair for $\Phi$, by $[9$, Definition 18]. This proves claim (ii).

The proof of claim (iii) is rather straightforward. First we note that because $\mathcal{D}$ has a $(J, S)$ inner-outer factorization with the outer part having a bounded inverse, it is by Lemma $22 J$-coercive. From [9, Lemma 18] we get the formula for the closed loop extended DLS $\Phi_{\diamond}^{e x t}:=[\Phi,[\mathcal{K}, \mathcal{F}]]_{\diamond}$

$$
\Phi_{\diamond}^{e x t}=\left[\begin{array}{cc}
A^{j}+\mathcal{B} \tau^{* j}(I-\mathcal{F})^{-1} \mathcal{K} & \mathcal{B} \tau^{* j}(I-\mathcal{F})^{-1} \\
{\left[\begin{array}{c}
\mathcal{C}+\mathcal{D}(I-\mathcal{F})^{-1} \mathcal{K} \\
(\mathcal{I}-\mathcal{F})^{-1} \mathcal{K}
\end{array}\right]} & {\left[\begin{array}{c}
\mathcal{D}(\mathcal{I}-\mathcal{F})^{-1} \\
(\mathcal{I}-\mathcal{F})^{-1}-I
\end{array}\right]}
\end{array}\right]
$$

By using the definitions of $\mathcal{K}, \mathcal{F}$, and the fact that $\mathcal{D}=\mathcal{N} \mathcal{X}$, we obtain from the previous

$$
\begin{aligned}
\Phi_{\diamond}^{\text {ext }} & =\left[\begin{array}{cl}
A^{j}-\mathcal{B} \mathcal{X}^{-1} \tau^{* j} S^{-1} \bar{\pi}_{+} \mathcal{N}^{*} J \mathcal{C} & \mathcal{B} \mathcal{X}^{-1} \tau^{* j} \\
{\left[\begin{array}{c}
\mathcal{C}-\mathcal{N} S^{-1} \bar{\pi}_{+} \mathcal{N}^{*} J \mathcal{C} \\
-\mathcal{X}^{-1} S^{-1} \bar{\pi}_{+} \mathcal{N}^{*} J \mathcal{C}
\end{array}\right]} & {\left[\begin{array}{c}
\mathcal{N} \\
\mathcal{X}^{-1}-\mathcal{I}
\end{array}\right]}
\end{array}\right] \\
& =\left[\begin{array}{cc}
\left(A^{\text {crit }}\right)^{j} & \mathcal{B} \mathcal{X}^{-1} \tau^{* j} \\
{\left[\begin{array}{l}
\mathcal{C}^{\text {crit }} \\
\mathcal{K}^{\text {crit }}
\end{array}\right]} & {\left[\begin{array}{c}
\mathcal{N} \\
\mathcal{X}^{-1}-\mathcal{I}
\end{array}\right]}
\end{array}\right],
\end{aligned}
$$

where the latter equality follows from Definition 7, claim (ii) of Lemma 8, claim (iii) of Lemma 22 and I/O-stability of $\Phi$. It is now clear, that this DLS outputs the critical signals with zero input, as desired. The I/O-stability of $\Phi_{\diamond}^{e x t}$ follows from the boundedness of $\mathcal{N}, \mathcal{X}^{-1}$ by Definition (18). Claim (iii) is now proved. The proof of the last part (iv) is trivial. This completes the proof of the lemma.

We remark that Proposition 21 gives a parameterization for the critical feedback pairs. In fact, all the critical feedback pairs are parameterized this way, because the previous lemma has the following converse:

Lemma 26. Let $\Phi=\left[\begin{array}{cc}A^{j} & \mathcal{B} \tau^{* j} \\ \mathcal{D} & \mathcal{D}\end{array}\right]$ be an I/O-stable $D L S$, and $J \in \mathcal{L}(Y)$ be self-adjoint. Let $[\mathcal{K}, \mathcal{F}]$ be an $I / O$-stable feedback pair for $\Phi$. Assume the following:

(i) $\Phi$ is J-coercive.

(ii) The critical control of $\Phi$ is of the feedback form with the I/O-stable feedback pair $[\mathcal{K}, \mathcal{F}]$.

Then the following holds:

(i) There exists a boundedly invertible $S \in \mathcal{L}(U)$ such that $(\mathcal{N}, \mathcal{X})$ is a $(J, S)$-innerouter factorization of $\mathcal{D}$, where

$$
\mathcal{X}:=\mathcal{I}-\mathcal{F},
$$




$$
\mathcal{N}:=\mathcal{D} \mathcal{X}^{-1}
$$

where $\mathcal{X}$ has bounded inverse.

(ii) The critical one step feedback operator satisfies $K^{\text {crit }} \in \mathcal{L}(H, U)$.

Proof. The main part of this proof lies in showing that claim (i) holds. Define $\mathcal{N}, \mathcal{X}$ as in equations (39) and (40). By using the closed loop formula for $\Phi_{\diamond}^{e x t}:=[\Phi,[\mathcal{K}, \mathcal{F}]]_{\diamond}$ and the fact that $\mathcal{D}=\mathcal{N} \mathcal{X}^{-1}$ in $\ell^{2}\left(\mathbf{Z}_{+} ; U\right)$, we obtain

$$
\begin{aligned}
& \Phi_{\diamond}^{e x t}=\left[\begin{array}{cl}
A^{j}-\mathcal{B} \mathcal{X}^{-1} \tau^{* j} \mathcal{K} & \mathcal{B X}^{-1} \tau^{* j} \\
{\left[\begin{array}{c}
\mathcal{C}+\mathcal{D} \mathcal{X}^{-1} \mathcal{K} \\
\mathcal{X}^{-1} \mathcal{K}
\end{array}\right]} & {\left[\begin{array}{c}
\mathcal{N} \\
\mathcal{X}^{-1}-\mathcal{I}
\end{array}\right]}
\end{array}\right] \\
& =\left[\begin{array}{cl}
\left(A^{\text {crit }}\right)^{j} & \mathcal{B X}^{-1} \tau^{* j} \\
{\left[\begin{array}{l}
\mathcal{C}^{\text {crit }} \\
\mathcal{K}^{\text {crit }}
\end{array}\right]} & {\left[\begin{array}{c}
\mathcal{N} \\
\mathcal{X}^{-1}-\mathcal{I}
\end{array}\right]}
\end{array}\right],
\end{aligned}
$$

where the latter equality follows from the assumption that the critical control of $\Phi$ is of the feedback form with the I/O-stable feedback pair $[\mathcal{K}, \mathcal{F}]$. See Definition 7 for the definitions of $\mathcal{K}^{\text {crit }}$ and $\mathcal{C}^{\text {crit }}$, and Lemma 8 for their basic properties.

We show now that $(\mathcal{N}, \mathcal{X})$ is a $(J, S)$-inner-outer factorization of $\mathcal{D}$ for some $S \in \mathcal{L}(U)$. By the definition of the operators $\mathcal{N}, \mathcal{X}$ we have $\mathcal{D}=\mathcal{N} \mathcal{X}$. Because $\Phi$ is I/O-stable and $J$-coercive, it is by Proposition 20 sufficient to show that $\mathcal{X}:=\mathcal{I}-\mathcal{F}$ is a $S$-spectral factor of $\mathcal{D}^{*} J \mathcal{D}$ for some $S \in \mathcal{L}(U)$. For this aim we try to find an invertible $S \in \mathcal{L}(U)$ satisfying

$$
\mathcal{D}^{*} J \mathcal{D}=\mathcal{X}^{*} S \mathcal{X}
$$

Let the operator $\mathcal{Z}$ be given by

$$
\mathcal{D}^{*} J \mathcal{N}=\mathcal{D}^{*} J \mathcal{D} \mathcal{X}^{-1}=\mathcal{X}^{*} S=: \mathcal{Z}^{*} .
$$

We show that $\mathcal{Z}^{*}:=\mathcal{D}^{*} J \mathcal{N}$ is anti-causal; i.e. $\bar{\pi}_{+} \mathcal{X}^{*} \pi_{-}=0$.

Let $\tilde{u} \in \operatorname{dom}(\mathcal{B})=\ell^{2}\left(\mathbf{Z}_{-} ; U\right) \cap \operatorname{Seq}(U)$ be arbitrary. Then $\pi_{-} \mathcal{X}^{-1} \pi_{-} \tilde{u} \in \operatorname{dom}(\mathcal{B})$ by causality, and we can set $x_{0}=\mathcal{B} \pi_{-} \mathcal{X}^{-1} \pi_{-} \tilde{u}=\mathcal{B} \mathcal{X}^{-1} \pi_{-} \tilde{u}$. We have $\operatorname{range}(\mathcal{B}) \subset \operatorname{dom}(\mathcal{C})$, by $\left[9\right.$, Lemma 39] and I/O-stability of $\Phi$, and consequently $x_{0} \in \operatorname{dom}(\mathcal{C}) \subset \operatorname{dom}\left(\mathcal{C}^{\text {crit }}\right)$. We can write for the critical control for DLS $\Phi$

$$
\tilde{y}^{\text {crit }}\left(x_{0}\right)=\mathcal{C}^{\text {crit }} x_{0}=\mathcal{C}^{\text {crit }}\left(\mathcal{B} \mathcal{X}^{-1}\right)=\bar{\pi}_{+} \mathcal{N} \pi_{-} \tilde{u}
$$

this holds because $\Phi_{\diamond}^{e x t}$ is the I/O-stable DLS of formula (41) outputting the critical control for $\Phi$ with zero control, and the fact that $\mathcal{C}^{\text {crit }}$ is the observability map and $\mathcal{B X}^{-1}$ is the controllability map for $\Phi_{\diamond}^{e x t}$.

Because $x_{0} \in \operatorname{dom}(\mathcal{C})$, we have by formula (10) of Lemma 4

$$
\bar{\pi}_{+} \mathcal{D}^{*} J \tilde{y}^{c r i t}\left(x_{0}\right)=0 .
$$


Now the equations (43) and (44) together give $\bar{\pi}_{+} \mathcal{D}^{*} J \mathcal{N} \pi_{-} \tilde{u}=\bar{\pi}_{+} \mathcal{Z}^{*} \pi_{-} \tilde{u}=0$ for all $\tilde{u} \in \operatorname{dom}(\mathcal{B})$. Because $\operatorname{dom}(\mathcal{B})$ is dense in $\ell^{2}\left(\mathbf{Z}_{-} ; U\right)$, and $\mathcal{X}^{*}$ is bounded, it follows that $\mathcal{Z}^{*}$ is anti-causal.

Because $\mathcal{D}^{*} J \mathcal{D}$ is self-adjoint, we have

$$
\mathcal{D}^{*} J \mathcal{D}=\mathcal{Z}^{*} \mathcal{X}=\mathcal{X}^{*} \mathcal{Z}
$$

or equivalently,

$$
\left(\mathcal{X}^{*}\right)^{-1} \mathcal{D}^{*} J \mathcal{D} \mathcal{X}^{-1}=\left(\mathcal{Z} \mathcal{X}^{-1}\right)^{*}=\mathcal{Z} \mathcal{X}^{-1}
$$

Because $\left(\mathcal{Z} \mathcal{X}^{-1}\right)^{*}$ is anti-causal and $\left(\mathcal{Z} \mathcal{X}^{-1}\right)$ is causal, it follows that $\left(\mathcal{X}^{*}\right)^{-1} \mathcal{D}^{*} J \mathcal{D} \mathcal{X}^{-1}$ is a static operator. Thus it is a multiplication by a self-adjoint operator $S \in \mathcal{L}(U)$. Clearly $S$ has a bounded inverse, because both $\mathcal{X}^{-1}$ and $\mathcal{Z}$ have; the latter requires the $J$-coercivity of $\mathcal{D}^{*} J \mathcal{D}$. So the existence of the required $S$ in equation (42) is established, and $(\mathcal{N}, \mathcal{X})$ is a $(J, S)$-inner-outer factorization of $\mathcal{D}$. This completes the proof of claim (i).

The remaining claim (ii) follows directly from the definition of the I/O-stable feedback pair, because $\mathcal{K}^{\text {crit }}=(\mathcal{I}-\mathcal{F})^{-1} \mathcal{K}$, by the closed loop formula (41). This completes the proof.

Now we are ready to present one of the main results of this paper. The next theorem tells us that under certain conditions, the spectral factorization problem of an I/O-map is equivalent with the problem of writing the critical minimax control of a DLS in the feedback form.

Theorem 27. Let $\Phi=\left[\begin{array}{cc}A^{j} & \mathcal{B} \tau^{* j} \\ \mathcal{C} & \mathcal{D}\end{array}\right]$ be an I/O-stable DLS, and $J \in \mathcal{L}(Y)$ be self-adjoint. Then the following conditions (i) and (ii) are equivalent:

(i) a) $\Phi$ is J-coercive,

b) There is an I/O-stable feedback pair $[\mathcal{K}, \mathcal{F}]$ for $\Phi$ such that the critical control of $\Phi$ is of the feedback form with the critical feedback pair equaling $[\mathcal{K}, \mathcal{F}]$.

(ii) a) There is a boundedly invertible operator $S \in \mathcal{L}(U)$ such that $\mathcal{D}$ has a $(J, S)$ inner-outer factorization $(\mathcal{N}, \mathcal{X})$, with the outer factor $\mathcal{X}$ having a bounded inverse,

b) $\pi_{0} \mathcal{N}^{*} J \mathcal{C} \in \mathcal{L}(H ; U)$.

Furthermore, if the above conditions hold, then both $\Phi^{\text {ext }}:=[\Phi,[\mathcal{K}, \mathcal{F}]]$ and $\Phi_{\diamond}^{\text {ext }}$ are I/O-stable.

Proof. (i) $\Rightarrow$ (ii) is a direct consequence of Lemma 26. In order to prove (ii) $\Rightarrow$ (i) we first note that if $\left(\mathcal{N}^{\prime}, \mathcal{X}^{\prime}\right)$ is a $\left(J, S^{\prime}\right)$-inner-outer factorization of $\mathcal{D}$ then by Proposition 21 there is a $(J, S)$-inner-outer factorization of $\mathcal{D}$ such that the static part satisfies $\pi_{0} \mathcal{X}^{-1} \pi_{0}=I$. 
Next we prove that $K^{\text {crit }}=\pi_{0} \mathcal{K}^{\text {crit }} \in \mathcal{L}(H, U)$. By claim (iii) of Lemma 22 and causality of $\mathcal{X}^{-1}$ we have

$$
K^{c r i t}=\pi_{0} \mathcal{K}^{c r i t}=-\pi_{0} \mathcal{X}^{-1} \pi_{0} \cdot \pi_{0} \mathcal{N}^{*} J \mathcal{C}
$$

Now $\pi_{0} \mathcal{X}^{-1} \pi_{0}$ is bounded with bounded inverse, by Proposition 19. It follows that $K^{\text {crit }}$ is boundedly invertible if and only if $\pi_{0} \mathcal{N}^{*} J \mathcal{C}$ is, giving part b) of (ii). Now an application of Lemma 25 completes the proof of the implication (ii) $\Rightarrow$ (i).

The following theorem states how the stabilities of the open and closed loop critical systems relate to each other. We remark that the output stability of an I/O-stable $J$ coercive $\Phi$ is a sufficient condition for the stability of the critical feedback pair $[\mathcal{K}, \mathcal{F}]$.

Theorem 28. Let $\Phi=\left[\begin{array}{cc}A_{\mathcal{C}}^{j} & \mathcal{B}_{\mathcal{D}}^{* j} \\ \mathcal{D}\end{array}\right]$ be an I/O-stable DLS, and let the feedback pair $[\mathcal{K}, \mathcal{F}]$ be stable. Then:

(i) $\Phi_{\diamond}^{e x t}$ is input stable if and only if $\Phi$ is.

(ii) $\Phi_{\diamond}^{e x t}$ is output stable if and only if $\Phi$ is.

(iii) $\Phi_{\diamond}^{e x t}$ is stable if and only if $\Phi$ is.

(iv) $\Phi_{\diamond}^{e x t}$ is strongly stable if $\Phi$ is.

Proof. See [9, Theorem 51].

\section{The Riccati equation system}

In the rest of this paper, a Riccati equation theory is developed for the minimax control problem introduced in Section 3. In the final Section 8, an equivalence result is given; we shall link together the Riccati equation theory of this section and Section 7 , and the critical feedback theory of the previous sections.

Let $J \in \mathcal{L}(Y)$ be self-adjoint and $\Phi=\left[\begin{array}{cc}A_{\mathcal{C}}^{j} & \mathcal{B} \tau^{* j} \\ \mathcal{D}\end{array}\right]$ be an I/O-stable $J$-coercive DLS. We show that the critical cost sesquilinear form $P^{c r i t}($, ), introduced in Definition 14, satisfies a Riccati equation system, provided that $\mathcal{D}$ has a $(J, S)$-inner-outer factorization. A converse result to this is given in section 7 .

Definition 29. Let $J \in \mathcal{L}(Y)$ be self-adjoint and $\phi=(\underset{C}{A} \underset{D}{B})$ be an I/O-stable $D L S$. Let $P($, ) denote a conjugate symmetric sesquilinear form, $P():, H \times H \supset \operatorname{dom}(\mathcal{C}) \times$ $\operatorname{dom}(\mathcal{C}) \rightarrow \mathbf{C}$. Then the conjugate symmetric sesquilinear form $\Lambda_{P}($,$) on U \times U$ defined by

$$
\Lambda_{P}\left(u_{0}, u_{1}\right):=\left\langle D u_{0}, J D u_{1}\right\rangle_{Y}+P\left(B u_{0}, B u_{1}\right)
$$

is called an indicator of the sesquilinear form $P($, ) (associated to $\Phi$ and $J$ ). 
The indicator $\Lambda_{P}($,$) is well defined on the whole of U \times U$. The possible problem would arise if we had to go outside the domain $\operatorname{dom}(\mathcal{C}) \times \operatorname{dom}(\mathcal{C})$ of $P($,$) for some u_{0} \in U$. However, by I/O-stability of $\Phi, B U \subset \operatorname{dom}(\mathcal{C})$ (see [9, Definition 37 and Lemma 40]). For the particular sesquilinear form $P^{\text {crit }}($, ) more can be said:

Proposition 30. Let $J \in \mathcal{L}(Y)$ be self-adjoint and $\Phi=\left(\begin{array}{cc}A & B \\ D\end{array}\right)$ be an I/O-stable $J$-coercive DLS. By $P^{\text {crit }}(,$,$) denote the critical cost quadratic form as defined in Definition 14. Then$ there exists a unique self adjoint operator $\Lambda_{P}^{\text {crit }} \in \mathcal{L}(U)$ such that the indicator $\Lambda_{P \text { crit }}($, satisfies

$$
\Lambda_{P^{c r i t}}\left(u_{0}, u_{1}\right)=\left\langle\Lambda_{P}^{c r i t} u_{0}, u_{1}\right\rangle_{U}
$$

where

$$
\Lambda_{P}^{\text {crit }}:=D^{*} J D+\left(\mathcal{C}^{\text {crit }} B\right)^{*} J\left(\mathcal{C}^{\text {crit }} B\right)
$$

Proof. The claim immediately follows, once we remember that by I/O-stability $\mathcal{C} B \in$ $\mathcal{L}(U, H)$. Then $\mathcal{K}^{\text {crit }} B$ is bounded by the definition of $\mathcal{K}^{\text {crit }}$, and so is $\mathcal{C}^{\text {crit }} B=\left(\mathcal{C}^{\text {crit }}+\mathcal{D} \mathcal{K}^{\text {crit }}\right) B$. This makes it possible to speak about $\left(\mathcal{C}^{\text {crit }} B\right)^{*}$ as an adjoint of a bounded operator. The self-adjointness and uniqueness of $\Lambda_{P}^{\text {crit }}$ is clear.

In the following lemma we couple $P^{\text {crit }}($,$) , the indicator \Lambda_{P}^{\text {crit }}$ and the critical one step feedback operator $K^{\text {crit }}:=\pi_{0} \mathcal{K}^{\text {crit }}$ together .

Lemma 31. Let $J \in \mathcal{L}(Y)$ be self-adjoint, and $\Phi=\left(\begin{array}{cc}A & B \\ C\end{array}\right)$ be an I/O-stable $J$-coercive $D L S$, such that $K^{\text {crit }}$ is bounded. Then $P^{\text {crit }}($, ) satisfies the equations

$$
\begin{gathered}
P^{c r i t}\left(A^{\text {crit }} x_{0}, B w_{0}\right)+\left\langle C^{c r i t} x_{0}, J D w_{0}\right\rangle_{Y}=0 \\
P^{c r i t}\left(A x_{0}, B w_{0}\right)+\left\langle\left(\Lambda_{P}^{c r i t} K^{c r i t}+D^{*} J C\right) x_{0}, w_{0}\right\rangle_{U}=0 \\
\Lambda_{P}^{c r i t} K^{c r i t} x_{0}=-\left(\left(\mathcal{C}^{c r i t} B\right)^{*} J \mathcal{C}^{c r i t} A-D^{*} J C\right) x_{0}
\end{gathered}
$$

for all $x_{0} \in \operatorname{dom}(\mathcal{C})$ and $w_{0} \in U$, and

$$
P^{c r i t}\left(A x_{0}, A x_{1}\right)-P^{c r i t}\left(x_{0}, x_{1}\right)=\left\langle\left(\left(K^{c r i t}\right)^{*} \Lambda_{P}^{c r i t} K^{c r i t}-C^{*} J C\right) x_{0}, x_{1}\right\rangle_{H}
$$

for all $x_{0}, x_{1} \in \operatorname{dom}(\mathcal{C})$, where $K^{\text {crit }}:=\pi_{0} \mathcal{K}^{\text {crit }}$ as in Definition $7, A^{\text {crit }}:=A+B K^{\text {crit }}$ as in Definition 10 and $C^{\text {crit }}:=C+D K^{\text {crit }}$.

Proof. In order to establish equation (46), we start with Frechet differentiating the identity

$$
\begin{aligned}
& J\left(x_{0}, \mathcal{K}^{c r i t} x_{0}+\epsilon\left(\pi_{0} \tilde{w}+\tau \mathcal{K}^{c r i t} B w_{0}\right)\right) \\
& =J\left(x_{0},\left(\pi_{0}\left(\tilde{u}^{c r i t}\left(x_{0}\right)+\epsilon \tilde{w}\right)+\tau \tilde{u}^{c r i t}\left(A x_{0}+B\left(u_{0}^{c r i t}\left(x_{0}\right)+\epsilon w_{0}\right)\right)\right)\right) \\
& =\left\langle C^{c r i t} x_{0}+\epsilon D w_{0}, J(-,,-)\right\rangle_{Y}+P^{c r i t}\left(A x_{0}+B\left(u_{0}^{c r i t}\left(x_{0}\right)+\epsilon w_{0}\right),(-,,-)\right)
\end{aligned}
$$

with respect to $\epsilon$ at $\epsilon=0$, where $\tilde{w}:=\left\{w_{j}\right\}_{j \geq 0}$. This derivative must equal zero, by the definition of $\mathcal{K}^{\text {crit }}$. We obtain the equality

$$
\operatorname{Re}\left(P^{c r i t}\left(A^{c r i t} x_{0}, B w_{0}\right)+\left\langle C^{c r i t} x_{0}, J D w_{0}\right\rangle_{Y}\right)=0 .
$$


This is as well true for the imaginary part, too. Equation (46) now follows.

The proof of equation (47) is based upon equation (46). We have by a straightforward calculation starting from the definition of $\Lambda_{P^{c r i t}}$

$$
\begin{aligned}
& \Lambda_{P^{c r i t}}\left(K^{c r i t} x_{0}, w_{0}\right):=P^{c r i t}\left(B K^{c r i t} x_{0}, B w_{0}\right)+\left\langle D K^{c r i t} x_{0}, J D w_{0}\right\rangle_{Y} \\
& =P^{c r i t}\left(A^{c r i t} x_{0}, B w_{0}\right)+\left\langle C^{c r i t} x_{0}, J D w_{0}\right\rangle_{Y} \\
& -P^{c r i t}\left(A x_{0}, B w_{0}\right)-\left\langle D^{*} J C x_{0}, w_{0}\right\rangle_{Y} .
\end{aligned}
$$

This proves that equation (47) is equivalent to equation (46).

Equation (48) follows immediately from equation (47) and the definition of $P^{\text {crit }}($, ). The proof of equation (49) is based on Lemma 11 and the first part of this lemma. Lemma 11 implies

$$
\begin{aligned}
& P^{c r i t}\left(A^{\text {crit }} x_{0}, A^{\text {crit }} x_{1}\right)=\left\langle\mathcal{C}^{c r i t} A^{\text {crit }} x_{0}, J \mathcal{C}^{c r i t} A^{\text {crit }} x_{1}\right\rangle_{\ell^{2}\left(\mathbf{Z}_{+} ; Y\right)} \\
& =\left\langle\bar{\pi}_{+} \tau^{*} \mathcal{C}^{c r i t} x_{0}, \bar{\pi}_{+} \tau^{*} J \mathcal{C}^{c r i t} x_{1}\right\rangle_{\ell^{2}\left(\mathbf{Z}_{+} ; Y\right)} \\
& =\left\langle\mathcal{C}^{c r i t} x_{0}, J \mathcal{C}^{c r i t} x_{1}\right\rangle_{\ell^{2}\left(\mathbf{Z}_{+} ; Y\right)}-\left\langle C^{c r i t} x_{0}, J C^{c r i t} x_{1}\right\rangle_{Y} \\
& =P^{c r i t}\left(x_{0}, x_{1}\right)-\left\langle C^{c r i t} x_{0}, J C^{c r i t} x_{1}\right\rangle_{Y} .
\end{aligned}
$$

A straightforward calculation, using $A^{\text {crit }}:=A+B K^{\text {crit }}$ and $C^{\text {crit }}:=C+D K^{\text {crit }}$, gives

$$
\begin{aligned}
& P^{c r i t}\left(A x_{0}, A x_{1}\right)-P^{c r i t}\left(x_{0}, x_{1}\right)+\left\langle C^{*} J C x_{0}, x_{1}\right\rangle_{H} \\
& =-P^{c r i t}\left(B K^{c r i t} x_{0}, A x_{1}\right)-\left\langle D K^{c r i t} x_{0}, J C x_{1}\right\rangle_{Y} \\
& -P^{c r i t}\left(A x_{0}, B K^{c r i t} x_{1}\right)-\left\langle J C x_{0}, D K^{c r i t} x_{1}\right\rangle_{Y} \\
& -P^{c r i t}\left(B K^{c r i t} x_{0}, B K^{c r i t} x_{1}\right)-\left\langle D K^{c r i t} x_{0}, J D K^{c r i t} x_{1}\right\rangle_{Y} \\
& =-\left[P^{c r i t}\left(B K^{c r i t} x_{0}, A^{c r i t} x_{1}\right)+\left\langle D K^{c r i t} x_{0}, J C^{c r i t} x_{1}\right\rangle_{Y}\right] \\
& +\left[P^{c r i t}\left(B K^{c r i t} x_{0}, B K^{c r i t} x_{1}\right)+\left\langle D K^{c r i t} x_{0}, J D K^{c r i t} x_{1}\right\rangle_{Y}\right] \\
& -\left[P^{c r i t}\left(A^{c r i t} x_{0}, B K^{c r i t} x_{1}\right)+\left\langle J C^{c r i t} x_{0}, D K^{c r i t} x_{1}\right\rangle_{Y}\right] \\
& +\left[P\left(B K^{c r i t} x_{0}, B K^{c r i t} x_{1}\right)-\left\langle D K^{c r i t} x_{0}, J D K^{c r i t} x_{1}\right\rangle_{Y}\right] \\
& -\Lambda_{P^{c r i t}}\left(K^{c r i t} x_{0}, K^{c r i t} x_{1}\right) \\
& =-\left[P^{c r i t}\left(B K^{c r i t} x_{0}, A^{c r i t} x_{1}\right)+\left\langle D K^{c r i t} x_{0}, J C^{c r i t} x_{1}\right\rangle_{Y}\right] \\
& {\left[P^{c r i t}\left(A^{c r i t} x_{0}, B K^{c r i t} x_{1}\right)+\left\langle J C^{c r i t} x_{0}, D K^{c r i t} x_{1}\right\rangle_{Y}\right]} \\
& +\Lambda_{P^{c r i t}}\left(K^{c r i t} x_{0}, K^{c r i t} x_{1}\right)+\Lambda_{P^{c r i t}}\left(K^{c r i t} x_{0}, K^{c r i t} x_{1}\right)-\Lambda_{P^{c r i t}}\left(K^{c r i t} x_{0}, K^{c r i t} x_{1}\right)
\end{aligned}
$$

Now an application of equation (46) with $K^{\text {crit }} x_{0}, K^{\text {crit }} x_{1}$ in place for $w_{0}$ completes the proof.

Under certain conditions, the indicator operator $\Lambda_{P}^{\text {crit }}$ has a bounded inverse. At the same time we get a connection between the $(J, S)$-inner-outer factorization of $\mathcal{D}$ and the indicator. This is the contents of the following lemma.

Lemma 32. Let $J \in \mathcal{L}(Y)$ be self-adjoint and $\Phi=\left[\begin{array}{cc}A_{\mathcal{C}}^{j} & \mathcal{B}_{\mathcal{D}}^{* j} \\ \mathcal{D}\end{array}\right]$ be an I/O-stable $J$-coercive $D L S$ such that $K^{\text {crit }}$ is bounded. By $P^{\text {crit }}($, ) denote the critical cost sesquilinear form. Assume that the conditions of Theorem 27 are satisfied. Then $\Lambda_{P}^{\text {crit }} \in \mathcal{L}(U)$ has a bounded inverse. 
Proof. Proving the case $u_{0}=u_{1}$ in sufficient. Choose any $\tilde{u}=\pi_{0} \tilde{u}$. Let $\Phi_{\diamond}^{e x t}$ be the critical closed loop DLS given by equation (41) of Theorem 27. Then

$$
\left(\begin{array}{c}
\mathcal{N} \\
\mathcal{X}^{-1}-\mathcal{I}
\end{array}\right) \tilde{u}=\left(\begin{array}{c}
N \\
X^{-1}-I
\end{array}\right) \pi_{0} \tilde{u}+\tau\left(\begin{array}{l}
\mathcal{C}^{c r i t} \\
\mathcal{K}^{c r i t}
\end{array}\right) B X^{-1} u_{0}
$$

by the basic properties of the $\Phi_{\diamond}^{e x t}$, where $u_{0}=\pi_{0} \tilde{u}$ with the identification of spaces range $\left(\pi_{0}\right)$ and $U$. Here $N:=\pi_{0} \mathcal{N} \pi_{0}$ and $X^{-1}:=\pi_{0} \mathcal{X}^{-1} \pi_{0}$, where $X, X^{-1} \in \mathcal{L}(U)$, by Proposition 19.

Equation (50) implies

$$
\begin{aligned}
& \left(\mathcal{X}^{-1}-\mathcal{I}\right) \tilde{u}=\left(X^{-1}-I\right) \pi_{0} \tilde{u}+\tau\left(\mathcal{K}^{c r i t} B\right) X^{-1} u_{0} \\
\Leftrightarrow \quad & \mathcal{X}^{-1} \pi_{0} \tilde{u}=X^{-1} \pi_{0} \tilde{u}+\tau \mathcal{K}^{c r i t} B X^{-1} u_{0}
\end{aligned}
$$

for all $\tilde{u}=\pi_{0} \tilde{u}$. By taking into consideration the assumed spectral factorization $\mathcal{D}^{*} J \mathcal{D}=$ $\mathcal{X}^{*} S \mathcal{X}$ (see Proposition 20), equation (51) implies

$$
\begin{aligned}
& J\left(0, X^{-1} \pi_{0} \tilde{u}+\tilde{u}^{c r i t}\left(B X^{-1} u_{0}\right)\right) \\
& =\left\langle\mathcal{D}^{*} J \mathcal{D}\left(X^{-1} \pi_{0} \tilde{u}+\tau \tilde{u}^{c r i t}\left(B X^{-1} u_{0}\right)\right),(-,,-)\right\rangle_{\ell^{2}\left(\mathbf{Z}_{+} ; U\right)} \\
& =\left\langle S \mathcal{X}\left(X^{-1} \pi_{0} \tilde{u}+\tau \tilde{u}^{c r i t}\left(B X^{-1} u_{0}\right)\right), \mathcal{X}(-,,-)\right\rangle_{\ell^{2}\left(\mathbf{Z}_{+} ; U\right)} \\
& =\left\langle S \mathcal{X}\left(\mathcal{X}^{-1} \pi_{0} \tilde{u}\right), \mathcal{X}\left(\mathcal{X}^{-1} \pi_{0} \tilde{u}\right)\right\rangle_{\ell^{2}\left(\mathbf{Z}_{+} ; U\right)}=\left\langle S u_{0}, u_{0}\right\rangle_{U}
\end{aligned}
$$

On the other hand, for all $\tilde{u}=\pi_{0} \tilde{u}$ we have

$$
\begin{aligned}
& J\left(0, X^{-1} \pi_{0} \tilde{u}+\tilde{u}^{\text {crit }}\left(B X^{-1} u_{0}\right)\right) \\
& =\left\langle D X^{-1} u_{0}, J D X^{-1} u_{0}\right\rangle_{Y}+P^{c r i t}\left(B X^{-1} u_{0}, B X^{-1} u_{0}\right)=: \Lambda_{P^{c r i t}}\left(X^{-1} u_{0}, X^{-1} u_{0}\right) .
\end{aligned}
$$

Now the combination of equations (52) and (53) gives

$$
\left\langle S u_{0}, u_{0}\right\rangle_{U}=\Lambda_{P^{c r i t}}\left(X^{-1} u_{0}, X^{-1} u_{0}\right)=\left(\left(X^{*}\right)^{-1} \Lambda_{P}^{c r i t} X^{-1} u_{0}, u_{0}\right)
$$

for all $u_{0} \in U$, where $S \in \mathcal{L}(U)$ is self-adjoint with bounded inverse. The last equality is by Proposition 30. Polarization ([18, Theorem 12.7]) implies now that $S=\left(X^{*}\right)^{-1} \Lambda_{P}^{\text {crit }} X^{-1}$. The claim of the lemma now follows, with $\Lambda_{P}^{\text {crit }}:=X^{*} S X$.

Now we have made sufficient preparations to approach the main result of this section, Lemma 35. We show that the $P^{\text {crit }}($, ) satisfies a Riccati equation system of the following type:

Definition 33. Let $J \in \mathcal{L}(Y)$ be self-adjoint, and $\Phi=\left[\begin{array}{c}A_{\mathcal{C}}^{j} \\ \mathcal{B}_{\mathcal{D}}^{* j}\end{array}\right]$ be an I/O-stable DLS. We say that the sesquilinear form $P($, , ) satisfies the Riccati equation system (associated to $J$ and $\Phi)$, if

$$
\begin{gathered}
P\left(A x_{0}, A x_{1}\right)-P\left(x_{0}, x_{1}\right)+\left(C^{*} J C x_{0}, x_{1}\right)_{H} \\
=\left\langle Q_{P}^{*} \Lambda_{P}^{-1} Q_{P} x_{0}, x_{1}\right\rangle_{H} \\
\left\langle\Lambda_{P} u_{0}, u_{1}\right\rangle=\left\langle D^{*} J D u_{0}, u_{1}\right\rangle_{U}+P\left(B u_{0}, B u_{1}\right) \\
\left\langle Q_{P} x_{1}, u_{2}\right\rangle=-\left\langle D^{*} J C x_{1}, u_{2}\right\rangle_{U}-P\left(A x_{1}, B u_{2}\right)
\end{gathered}
$$

for all $u_{0}, u_{1}, u_{2} \in U$ and $x_{0}, x_{1}, x_{2} \in \operatorname{dom}(\mathcal{C})$, where the linear operators satisfy $\Lambda_{P}, \Lambda_{P}^{-1} \in$ $\mathcal{L}(U)$ and $Q_{P} \in \mathcal{L}(H ; U)$. 
Given a self-adjoint $J \in \mathcal{L}(Y)$ and an I/O-stable $\Phi=\left[\begin{array}{c}A^{j} \mathcal{B}_{\mathcal{T}}^{* * j} \\ \mathcal{C}\end{array}\right]$, the Riccati equation system (54) - (56) in general has a plenty of solutions, of which only few are control theoretically interesting. In the following definition we give a tool that can be used to separate the interesting solutions from the non-interesting ones.

Definition 34. Let $J \in \mathcal{L}(Y)$ be self-adjoint, and $\Phi=\left[\begin{array}{cc}A_{\mathcal{C}}^{j} & \mathcal{B}_{\mathcal{T}}^{* j} \\ \mathcal{D}\end{array}\right]$ be an I/O-stable DLS. Let $P($, , ) be any conjugate symmetric solution of the Riccati equation system (54) - (56). Then the DLS

$$
\phi_{P}:=\left(\begin{array}{cc}
A & B \\
-Q_{P} & \Lambda_{P}
\end{array}\right)
$$

is called the indicator DLS (associated to $J$ and $\Phi$ ) of the sesquilinear form $P($, ), where the bounded linear operators $Q_{P}, \Lambda_{P}$ are as in Definition 33.

Now the main result of this section:

Lemma 35. Let $J \in \mathcal{L}(Y)$ be self-adjoint, and $\Phi=\left[\begin{array}{cc}A^{j} & \mathcal{B}_{\mathcal{C}}^{* j} \\ \mathcal{C}\end{array}\right]$ be an I/O-stable DLS. Moreover, assume that the equivalent conditions of Theorem 27 are satisfied. Then the following holds:

(i) $P^{\text {crit }}($, ) satisfies the Riccati equation system (54)-(56) of Definition 33.

(ii) For all $x_{0} \in \operatorname{dom}(\mathcal{C}), \tilde{u} \in \ell^{2}\left(\mathbf{Z}_{+} ; U\right): P^{\text {crit }}\left(x_{k}\left(x_{0}, \tilde{u}\right), x_{k}\left(x_{0}, \tilde{u}\right),\right) \rightarrow 0$ as $k \rightarrow \infty$.

(iii) The indicator DLS $\phi_{\text {Pcrit }}$ is both I/O-stable and outer with bounded inverse.

To say that $\phi_{P}$ is outer means that the I/O-map of $\phi_{P^{c r i t}}$ is outer in the sense of Definition 18.

Proof. Let us first look at the solutions of equations (55) and (56) if we have $P^{\text {crit }}()=$, $P($, ). By Definition 29, Proposition 30 and Lemma 32 there is an unique self-adjoint boundedly invertible operator, the critical indicator $\Lambda_{P}^{\text {crit }}$, solving equation (55) with $P^{\text {crit }}(,$,$) in the place of P($,$) . Equation (47) of Lemma 31$ implies that $Q_{P \text { crit }}:=\Lambda_{P}^{\text {crit }} K^{\text {crit }}$ satisfies equation (56). By the conditions of Theorem 27, $K^{\text {crit }}$ is bounded and so is $Q_{P \text { crit }}$.

Now that we know what the operators $\Lambda_{P \text { crit }}$ and $Q_{P \text { crit }}$ are, we still have to check that equation (54) holds with $P^{\text {crit }}($, , ) in the place of $P($,$) . This follows from equation 49$ of Lemma 31. This completes the proof of claim (i).

Claim (ii) is a direct consequence of Proposition 15. It remains to show claim (iii) stating that the indicator DLS $\phi_{P^{c r i t}}=\left(\begin{array}{cc}A & B \\ -Q_{P c r i t} & \Lambda_{P c r i t}\end{array}\right)$ is both I/O-stable and outer with bounded inverse. Equivalently, we must show that $\left(\begin{array}{cc}A & B \\ K^{c r i t} & -I\end{array}\right)$ is both I/O-stable and outer with bounded inverse, because $\left(\Lambda_{P^{c r i t}}\right)^{-1}=\left(\Lambda_{P}^{\text {crit }}\right)^{-1}$ is bounded by Lemma 32 , and $K^{\text {crit }}=$ $\left(\Lambda_{P}^{c r i t}\right)^{-1} Q_{P \text { crit }}$.

Because the conditions of Theorem 27 hold, we have a $(J, S)$-inner-outer factorization $(\mathcal{N}, \mathcal{X})$ of $\mathcal{D}$ for some $S$. By Proposition 21, we can find another $(J, S)$-inner-outer 
factorization, say $\left(\mathcal{N}^{\prime}, \mathcal{X}^{\prime}\right)$, such that $\pi_{0} \mathcal{X}^{\prime} \pi_{0}=I$. Use this factorization to construct a critical I/O-stable feedback pair $[\mathcal{K}, \mathcal{F}]$ in I/O-form by formulae $(37)$.

Now we have $\pi_{0} \mathcal{F} \pi_{0}=0$ because $\mathcal{X}=\mathcal{I}-\mathcal{F}$. When writing $[\mathcal{K}, \mathcal{F}]$ in the difference equation form, we obtain $\left(K^{\text {crit }}, 0\right)$. But then the I/O-map of $\phi_{P^{c r i t}}=\left(\begin{array}{c}A \\ K^{c r i t}\end{array}-I\right)$ equals $\mathcal{F}-\mathcal{I}$. It follows that $\phi_{\text {Prit }}$ is I/O-stable and outer with a bounded inverse because both the operators $\mathcal{I}-\mathcal{F}$ and $(\mathcal{I}-\mathcal{F})^{-1}$ are bounded, causal and shift invariant, by the definition of the I/O-stable feedback pair $[\mathcal{K}, \mathcal{F}]$. This completes the proof.

\section{$7 \quad$ Solution of the Riccati equation system}

In this section we give a converse for Lemma 31. We show that if the Riccati equation system (54) - (56) of Definition 33 has a solution of a special kind, then the conditions of Theorem 27 are satisfied. The speciality of the solution is in the requirement that the indicator DLS $\phi_{P}$, must be both I/O-stable and outer with a bounded inverse. We start with a fairly technical preliminary proposition.

Proposition 36. Let $\Phi$ be an I/O-stable DLS. Let $P($, ) be a solution of Riccati equation system (54)- (56). Let $\tilde{u} \in \ell^{2}\left(\mathbf{Z}_{+} ; U\right), x_{0} \in \operatorname{dom}(\mathcal{C})$ be arbitrary. Then

(i) for $u_{k}=u_{k}\left(x_{0}, \tilde{u}\right)$ and $x_{k}=x_{k}\left(x_{0}, \tilde{u}\right)$ we have

$$
\begin{aligned}
& P\left(x_{k}, x_{k}\right)-P\left(x_{k+1}, x_{k+1}\right) \\
& =\left\langle J\left(C x_{k}+D u_{k}\right),(-,,-)\right\rangle_{Y}-\left\langle\Lambda_{P}^{-1}\left(-Q_{P} x_{k}+\Lambda_{P} u_{k}\right),(-,,-)\right\rangle_{U},
\end{aligned}
$$

and for all $n \geq 1$

$$
\begin{aligned}
& P\left(x_{0}, x_{0}\right)-P\left(x_{n+1}, x_{n+1}\right) \\
& =\sum_{k=0}^{n}\left\langle J\left(C x_{k}+D u_{k}\right),(-,,-)\right\rangle_{Y} \\
& -\sum_{k=0}^{n}\left\langle\Lambda_{P}^{-1}\left(-Q_{P} x_{k}+\Lambda_{P} u_{k}\right),(-,,-)\right\rangle_{U} .
\end{aligned}
$$

(ii) If, in addition, $P\left(x_{k}\left(x_{0}, \tilde{u}\right), x_{k}\left(x_{0}, \tilde{u}\right)\right) \rightarrow 0$ as $k \rightarrow \infty$ for all $\tilde{u} \in \ell^{2}\left(\mathbf{Z}_{+} ; U\right)$ and $x_{0} \in \operatorname{dom}(\mathcal{C})$, then

$$
J\left(x_{0}, \tilde{u}\right)=P\left(x_{0}, x_{0}\right)+\sum_{k=0}^{\infty}\left\langle\Lambda_{P}^{-1}\left(-Q_{P} x_{k}+\Lambda_{P} u_{k}\right),(-,,-)\right\rangle_{U},
$$

where the sum converges.

(iii) If, in addition, $\phi_{P}$ is I/O-stable, and $P\left(x_{k}\left(x_{0}, \tilde{u}\right), x_{k}\left(x_{0}, \tilde{u}\right)\right) \rightarrow 0$ as $k \rightarrow \infty$ for all $\tilde{u} \in \ell^{2}\left(\mathbf{Z}_{+} ; U\right)$ and $x_{0} \in \operatorname{dom}(\mathcal{C})$, then

$$
J\left(x_{0}, \tilde{u}\right)=P\left(x_{0}, x_{0}\right)+\left\langle\Lambda_{P}^{-1}\left(\mathcal{C}_{\phi_{P}} x_{0}+\mathcal{D}_{\phi_{P}} \tilde{u}\right),(-,,-)\right\rangle_{\ell^{2}\left(\mathbf{Z}_{+} ; U\right)}
$$

for all $x_{0} \in \operatorname{dom}(\mathcal{C}) \cap \operatorname{dom}\left(\mathcal{C}_{\phi_{P}}\right)$. 
Proof. Claim (i) is proved calculating

$$
\begin{aligned}
& P\left(x_{k}, x_{k}\right)-P\left(x_{k+1}, x_{k+1}\right)=P\left(x_{k}, x_{k}\right)-P\left(A x_{k}+B u_{k}, A x_{k}+B u_{k}\right) \\
& =P\left(x_{k}, x_{k}\right)-P\left(A x_{k}, A x_{k}\right)-P\left(A x_{k}, B u_{k}\right)-P\left(B u_{k}, A x_{k}\right)-P\left(B u_{k}, B u_{k}\right) .
\end{aligned}
$$

Because $P($, ) satisfies the Riccati equation system (54)-(56), the previous equals:

$$
\begin{aligned}
& =\left\langle\left(C^{*} J C-Q_{P}^{*} \Lambda_{P}^{-1} Q_{P}\right) x_{k}, x_{k}\right\rangle_{H} \\
& +\left\langle\left(Q_{P}+D^{*} J C\right) x_{k}, u_{k}\right\rangle_{U}+\left\langle u_{k},\left(Q_{P}+D^{*} J C\right) x_{k}\right\rangle_{U} \\
& +\left\langle\left(D^{*} J D-\Lambda_{P}\right) u_{k}, u_{k}\right\rangle_{U} \\
& =\left[\left\langle C^{*} J C x_{k}, x_{k}\right\rangle_{H}+\left\langle D^{*} J C x_{k}, u_{k}\right\rangle_{U}+\left\langle u_{k}, D^{*} J C x_{k}\right\rangle_{U}+\left\langle D^{*} J D u_{k}, u_{k}\right\rangle_{U}\right] \\
& +\left[\left\langle-Q_{P}^{*} \Lambda_{P}^{-1} Q_{P} x_{k}, x_{k}\right\rangle_{H}+\left\langle Q_{P} x_{k}, u_{k}\right\rangle_{U}+\left\langle u_{k}, Q_{P} x_{k}\right\rangle_{U}-\left\langle\Lambda_{P} u_{k}, u_{k}\right\rangle_{U}\right] \\
& =\left\langle J\left(C x_{k}+D u_{k}\right),(-,,-)\right\rangle_{Y}-\left\langle\Lambda_{P}^{-1}\left(-Q_{P} x_{k}+\Lambda_{P} u_{k}\right),(-,,-)\right\rangle_{U}
\end{aligned}
$$

where the last equality is obtained simply by grouping terms. This proves equation (58). Equation (59) is now an immediate consequence.

Claim (ii) is proved by inspection of equation (59). We have for each $n \geq 1$

$$
\begin{aligned}
& \sum_{k=0}^{n}\left\langle\Lambda_{P}^{-1}\left(-Q_{P} x_{k}+\Lambda_{P} u_{k}\right),(-,,-)\right\rangle_{U} \\
& =-P\left(x_{0}, x_{0}\right)+P\left(x_{n+1}, x_{n+1}\right)+\sum_{k=0}^{n}\left\langle J\left(C x_{k}+D u_{k}\right),(-,,-)\right\rangle_{Y}
\end{aligned}
$$

Now the sum in the right hand side converges absolutely as $n \rightarrow \infty$, because $\Phi$ is I/Ostable, $x_{0} \in \operatorname{dom}(\mathcal{C})$ and $\tilde{u} \in \ell^{2}\left(\mathbf{Z}_{+} ; U\right)$. By assumption, also $P\left(x_{n+1}, x_{n+1}\right) \rightarrow 0$ as $n \rightarrow \infty$. It follows that $\lim _{n \rightarrow \infty} \sum_{k=0}^{n}\left\langle\Lambda_{P}^{-1}\left(-Q_{P} x_{k}+\Lambda_{P} u_{k}\right),(-,,-)\right\rangle_{U}$ exists and satisfies $(60)$.

In order to prove the final claim (iii), note that the I/O-stability of $\phi_{P}$ implies that the sequence $\left\{-Q_{P} x_{k}+\Lambda_{P} u_{k}\right\}_{k \geq 0}=\mathcal{C}_{\phi_{P}} x_{0}+\mathcal{D}_{\phi_{P}} \tilde{u} \in \ell^{2}\left(\mathbf{Z}_{+} ; U\right)$, if $x_{0} \in \operatorname{dom}\left(\mathcal{C}_{\phi_{P}}\right)$ and $\tilde{u} \in \ell^{2}\left(\mathbf{Z}_{+} ; U\right)$. Then the sum in (60) represents an inner product of two $\ell^{2}\left(\mathbf{Z}_{+} ; U\right)$ sequences, and thus converges absolutely.

Note that the intersection $\operatorname{dom}(\mathcal{C}) \cap \operatorname{dom}\left(\mathcal{C}_{\phi_{P}}\right)$ in claim (iii) of Proposition 36 is far from empty for I/O-stable $\Phi, \phi_{P}$. In particular, because $\mathcal{B}=\mathcal{B}_{\phi_{P}}$, and for I/O-stable systems always range $(\mathcal{B}) \subset \operatorname{dom}(\mathcal{C})$, it follows that range $(\mathcal{B}) \subset \operatorname{dom}(\mathcal{C}) \cap \operatorname{dom}\left(\mathcal{C}_{\phi_{P}}\right) . \quad \mathrm{A}$ crucial connection between a solution of Riccati equation system and a certain factorization of the Popov operator is given below.

Lemma 37. Let $J \in \mathcal{L}(Y)$ be self-adjoint, and $\Phi=\left[\begin{array}{cc}A_{\mathcal{C}}^{j} & \mathcal{B}_{\mathcal{D}}^{* j} \\ \mathcal{D}\end{array}\right]$ be an I/O-stable DLS. Let $P($, ) be the solution of the Riccati equation system (54)-(56) of Definition 33 such that the indicator DLS $\phi_{P}$ is I/O-stable and $P\left(x_{k}\left(x_{0}, \tilde{u}\right), x_{k}\left(x_{0}, \tilde{u}\right)\right) \rightarrow 0$ as $k \rightarrow \infty$ for all $\tilde{u} \in \ell^{2}\left(\mathbf{Z}_{+} ; U\right)$ and $x_{0} \in \operatorname{dom}(\mathcal{C})$. Then there is a factorization

$$
\mathcal{D}^{*} J \mathcal{D}=\mathcal{D}_{\phi_{P}}^{*} \Lambda_{P}^{-1} \mathcal{D}_{\phi_{P}}
$$


Proof. The both sides of (62) are bounded, causal and shift invariant operators. We prove that their Toeplitz operators are equal. For all $\tilde{u} \in \ell^{2}\left(\mathbf{Z}_{+} ; U\right)$ we have

$$
J(0, \tilde{u})=\left\langle\mathcal{D}^{*} J \mathcal{D} \tilde{u}, \tilde{u}\right\rangle_{\ell^{2}\left(\mathbf{Z}_{+} ; U\right)} .
$$

By linearity of $P\left(x_{0}, x_{1}\right)$ in $x_{0}$ we get $P(0,0)=0$. Then by claim (iii) of Proposition 36 we have for all $\tilde{u} \in \ell^{2}\left(\mathbf{Z}_{+} ; U\right)$

$$
J(0, \tilde{u})=\left\langle\mathcal{D}_{\phi_{P}}^{*} \Lambda_{P}^{-1} \mathcal{D}_{\phi_{P}} \tilde{u}, \tilde{u}\right\rangle_{\ell^{2}\left(\mathbf{Z}_{+} ; U\right)} .
$$

By combining equations (63) and (64), and noting that $\bar{\pi}_{+}\left(\mathcal{D}^{*} J \mathcal{D}-\mathcal{D}_{\phi_{P}}^{*} \Lambda_{P}^{-1} \mathcal{D}_{\phi_{P}}\right) \bar{\pi}_{+}$is self-adjoint, we conclude equation (62) from

$$
\left\langle\left(\mathcal{D}^{*} J \mathcal{D}-\mathcal{D}_{\phi_{P}}^{*} \Lambda_{P}^{-1} \mathcal{D}_{\phi_{P}}\right) \tilde{u}, \tilde{u}\right\rangle_{\ell^{2}\left(\mathbf{Z}_{+} ; U\right)}=0
$$

because $\tilde{u} \in \ell^{2}\left(\mathbf{Z}_{+} ; U\right)$ was arbitrary. This completes the proof.

If $P($,$) has the special property such that the indicator DLS \phi_{P}$ is, in addition, outer with bounded inverse, the the factorization of Lemma 37 can be put in a more familiar form:

Corollary 38. Let $J \in \mathcal{L}(Y)$ be self-adjoint, and $\Phi=\left[\begin{array}{c}A_{\mathcal{C}}^{j} \mathcal{B}_{\mathcal{D}}^{* j} \\ \mathcal{D}\end{array}\right]$ be an I/O-stable DLS. Let $P($,$) be the solution of the Riccati equation system (54)-(56) of Definition 33$ such that the indicator DLS $\phi_{P}$ is I/O-stable and outer with a bounded inverse, and $P^{\text {crit }}\left(x_{k}\left(x_{0}, \tilde{u}\right), x_{k}\left(x_{0}, \tilde{u}\right),\right) \rightarrow 0$ as $k \rightarrow \infty$ for all $x_{0} \in \operatorname{dom}(\mathcal{C}), \tilde{u} \in \ell^{2}\left(\mathbf{Z}_{+} ; U\right)$. Then $\mathcal{D}$ has a $\left(J, \Lambda_{P}\right)$-inner-outer factorization $\left(\mathcal{N}_{P}, \mathcal{X}_{P}\right)$

$$
\begin{aligned}
\mathcal{N}_{P} & :=\mathcal{D D}_{\phi_{P}}^{-1} \Lambda_{P} \\
\mathcal{X}_{P} & :=\Lambda_{P}^{-1} \mathcal{D}_{\phi_{P}},
\end{aligned}
$$

where the outer part $\mathcal{X}_{P}$ has a bounded inverse.

Proof. The claim trivially follows from equation (62).

Note that the I/O-map of the indicator DLS $\phi_{P}$ is a spectral factor of $\mathcal{D}$, when the conditions of Corollary 38 are met. The previous results are collected in the following lemma, the main result of this section. It is the converse for Lemma 31.

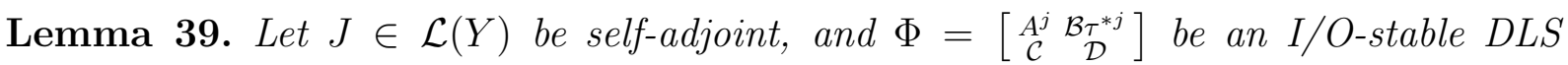
satisfying $\overline{\text { range }(\mathcal{B})}=H$. Assume the following:

(i) The sesquilinear form $P($, ) satisfies the Riccati equation system (54)-(56) of Definition 33.

(ii) For all $x_{0} \in \operatorname{dom}(\mathcal{C}), \tilde{u} \in \ell^{2}\left(\mathbf{Z}_{+} ; U\right) P^{\text {crit }}\left(x_{k}\left(x_{0}, \tilde{u}\right), x_{k}\left(x_{0}, \tilde{u}\right)\right) \rightarrow 0$ as $k \rightarrow \infty$.

(iii) The indicator DLS $\phi_{P}$ is both I/O-stable and outer with a bounded inverse. 
Then the conditions of Theorem 27 are satisfied.

Proof. We construct a critical I/O-stable feedback pair from the factorization $\left(\mathcal{N}_{P}, \mathcal{X}_{P}\right)$ of Corollary 38. We start with writing the indicator DLS $\phi_{P}$ in I/O-form

$$
\phi_{P}=\left(\begin{array}{cc}
A & B \\
-Q_{P} & \Lambda_{P}
\end{array}\right)=\left[\begin{array}{cc}
A^{j} & \mathcal{B} \tau^{* j} \\
\mathcal{K}_{P} & \mathcal{I}-\mathcal{F}_{P}
\end{array}\right]
$$

This defines the operators $\mathcal{K}_{P}$ and $\mathcal{F}_{P}$. Because $\phi_{P}$ is I/O-stable and outer with a bounded inverse, it follows that $\left[\mathcal{K}_{P}, \mathcal{F}_{P}\right]$ is an I/O-stable feedback pair for $\Phi$. We need one more operator

$$
\mathcal{K}_{P}^{\prime}:=-\bar{\pi}_{+} \mathcal{N}_{P}^{*} J \mathcal{C}
$$

$\mathcal{K}^{\prime}$ is a linear operator from $\operatorname{dom}(\mathcal{C}) \rightarrow \ell^{2}\left(\mathbf{Z}_{+} ; U\right)$. A similar calculation as in the proof of Lemma 25 implies that $\mathcal{K}_{P}^{\prime} A=\bar{\pi}_{+} \tau^{*} \mathcal{K}_{P}^{\prime}$ in $\operatorname{dom}(\mathcal{C})$, and $\mathcal{K}_{P}^{\prime} \mathcal{B}=\bar{\pi}_{+}\left(\mathcal{I}-\mathcal{F}_{P}\right) \pi_{-}$in $\operatorname{dom}(\mathcal{B})$. If we can show that $K_{P}^{\prime}:=\pi_{0} \mathcal{K}_{P}^{\prime}: \operatorname{dom}(\mathcal{C}) \rightarrow U$ is bounded, we can extend it continuously to the whole of $H$, because $\overline{\operatorname{dom}(\mathcal{C})}=H$. This would make the system

$$
\left[\begin{array}{cc}
A^{j} & \mathcal{B} \tau^{* j} \\
\mathcal{K}_{P}^{\prime} & \mathcal{I}-\mathcal{F}_{P}
\end{array}\right]
$$

an I/O-stable DLS whose I/O-map is outer with a bounded inverse. Furthermore,

$$
\mathcal{K}^{\text {crit }}=\left(\mathcal{I}-\mathcal{F}_{P}\right)^{-1} \mathcal{K}_{P}^{\prime}
$$

which is proved by applying the $\left(J, \Lambda_{P}\right)$-inner-outer factorization $\left(\mathcal{N}_{P}, \mathcal{X}_{P}\right)$ on the formula for $\mathcal{K}^{\text {crit }}$ in claim (iii) of Lemma 22 , and noting that $\mathcal{I}-\mathcal{F}_{P}=\Lambda_{P} \mathcal{X}_{P}$.

The systems in equations (65) and (66) are remarkably similar. We know that $\left[\mathcal{K}_{P}, \mathcal{F}_{P}\right]$ is an I/O-stable feedback pair for $\Phi$ but we do not know whether it is critical. We do not know that $\left[\mathcal{K}_{P}^{\prime}, \mathcal{F}_{P}\right]$ is a feedback pair but if it is, then it is critical, by equation (67). We complete the proof by showing that the operators $\pi_{0} \mathcal{K}_{P}^{\prime}, \pi_{0} \mathcal{K}_{P}$ coincide.

Because the I/O-maps of the systems (65) and (66) are equal, we can write for each $\tilde{u} \in \operatorname{dom}(\mathcal{B})$

$$
\pi_{0} \mathcal{K}_{P}(\mathcal{B} \tilde{u})=\pi_{0}\left(\mathcal{I}-\mathcal{F}_{P}\right) \pi_{-} \tilde{u}=\pi_{0} \mathcal{K}_{P}^{\prime}(\mathcal{B} \tilde{u}) .
$$

Because the operator $\pi_{0} \mathcal{K}_{P}=-Q_{P}$ is bounded in the topology of $H$ by Definition 33, and $\overline{\text { range }(\mathcal{B})}=H$, it follows that also $K_{P}^{\prime}=\pi_{0} \mathcal{K}_{P}^{\prime}:$ range $(\mathcal{B}) \rightarrow U$ is densely defined and bounded in $H . K_{P}^{\prime}$ can now be identified with its bounded extension to the whole of $H$. This proves that the system (66) is a DLS and furthermore $\mathcal{K}_{P}=\mathcal{K}_{P}^{\prime}$. This completes the proof.

\section{Equivalence results for I/O-stable DLS's}

The main theorem of this paper is a conclusion of Theorem 27 and Lemmas 31, 39. 
Theorem 40. Let $\Phi=\left[\begin{array}{cc}A^{j} & \mathcal{B} \tau^{* j} \\ \mathcal{C} & \mathcal{D}\end{array}\right]$ be an I/O-stable DLS, and $J \in \mathcal{L}(Y)$ be self-adjoint. Assume that $\overline{\text { range }(\mathcal{B})}=H$. Then the following conditions (i), (ii) and (iii) are equivalent:

(i) a) $\Phi$ is J-coercive.

b) There is an I/O-stable feedback pair $[\mathcal{K}, \mathcal{F}]$ for $\Phi$ such that the critical control of $\Phi$ is of feedback form with the critical feedback pair $[\mathcal{K}, \mathcal{F}]$.

(ii) a) There is a boundedly invertible operator $S \in \mathcal{L}(U)$ such that $\mathcal{D}$ has a $(J, S)$ inner-outer factorization $(\mathcal{N}, \mathcal{X})$ with the outer part $\mathcal{X}$ having a bounded inverse.

b) $\pi_{0} \mathcal{N}^{*} J \mathcal{C} \in \mathcal{L}(H ; U)$.

(iii) There is a solution $P()=,P^{c r i t}($, ) of the Riccati equation system (54)-(56) satisfying

a) The indicator DLS $\phi_{P}$ is both I/O-stable and outer with a bounded inverse.

b) $P\left(x_{k}\left(x_{0}, \tilde{u}\right), x_{k}\left(x_{0}, \tilde{u}\right)\right) \rightarrow 0$ as $k \rightarrow \infty$ for all trajectories of $\Phi$ with $x_{0} \in$ $\operatorname{dom}(\mathcal{C})$ and $\tilde{u} \in \ell^{2}\left(\mathbf{Z}_{+} ; U\right)$.

When the equivalent conditions (i), (ii) and (iii) hold, then

- the corresponding $[\mathcal{K}, \mathcal{F}]$ and $(\mathcal{N}, \mathcal{X})$ are related by formulae (37), (39) and (40),

- the corresponding $(\mathcal{N}, \mathcal{X})$ and $P($, ) are related by Definition 34 and Corollary 38,

- the corresponding $P($,$) and [\mathcal{K}, \mathcal{F}]$ are related as in the proof of Lemma 39.

In the light of claim (iii) of Theorem 40, conditions for the I/O-stability of $\phi_{P}$ in terms of $P($,$) would be useful. We remind that for I/O-stable and J$-coercive $\Phi$, $P^{\text {crit }}\left(x_{k}\left(x_{0}, \tilde{u}\right), x_{k}\left(x_{0}, \tilde{u}\right)\right) \rightarrow 0$ for $x_{0} \in \operatorname{dom}(\mathcal{C}), \tilde{u} \in \ell^{2}\left(\mathbf{Z}_{+} ; U\right)$, by Proposition 15 . An additional speed estimate for this convergence speed is the key observation.

Proposition 41. Let $\Phi$ be an I/O-stable DLS. Let $P($, ) be a solution of Riccati equation system (54)-(56) such that $P\left(x_{k}\left(x_{0}, \tilde{u}\right), x_{k}\left(x_{0}, \tilde{u}\right)\right) \rightarrow 0$ for all $\tilde{u} \in \ell^{2}\left(\mathbf{Z}_{+} ; U\right)$ and $x_{0} \in$ $\operatorname{dom}(\mathcal{C})$. Then $\phi_{P}$ is I/O-stable if and only if

$$
\sum_{k=0}^{\infty}\left|P\left(x_{k}, x_{k}\right)-P\left(x_{k+1}, x_{k+1}\right)\right|<\infty
$$

for all $\tilde{u} \in \ell^{2}\left(\mathbf{Z}_{+} ; U\right)$ and $x_{0} \in \operatorname{dom}(\mathcal{C})$, where $x_{k}=x_{k}\left(x_{0}, \tilde{u}\right)$.

Proof. For any self-adjoint, boundedly invertible operator $T$ in a Hilbert space, the following estimate holds:

$$
\left\|T^{-1}\right\|^{-1}\langle x, x\rangle \leq|\langle T x, x\rangle| \leq\|T\|\langle x, x\rangle
$$


Applying this with $T=\Lambda_{P}^{-1}$ gives the equivalence:

$$
\sum_{k=0}^{\infty}\left|\left\langle\Lambda_{P}^{-1}\left(-Q_{P} x_{k}+\Lambda_{P} u_{k}\right),(-,,-)\right\rangle_{U}\right|<\infty
$$

if and only if

$$
\sum_{k=0}^{\infty}\left|\left\langle\left(-Q_{P} x_{k}+\Lambda_{P} u_{k}\right),(-,,-)\right\rangle_{U}\right|=\left\|\left\{-Q_{P} x_{k}+\Lambda_{P} u_{k}\right\}\right\|_{\ell^{2}\left(\mathbf{Z}_{+} ; U\right)}^{2}<\infty .
$$

We first show that first (69) is equivalent with the boundedness of $\mathcal{D}_{\phi_{P}} \bar{\pi}_{+}$; i.e. I/Ostability of $\phi_{P}$. Now let $\tilde{u} \in \ell^{2}\left(\mathbf{Z}_{+} ; U\right)$ be arbitrary and $x_{k}=x_{k}(0, \tilde{u})=\mathcal{B}_{\phi_{P}} \tau^{* j} \tilde{u}=\mathcal{B} \tau^{* j} \tilde{u}$. Then $\left\{-Q_{P} x_{k}+\Lambda_{P} u_{k}\right\}_{k \geq 0}=\mathcal{D}_{\phi_{P}} \bar{\pi}_{+} \tilde{u}$. It follows that (69) holds for all $\tilde{u} \in \ell^{2}\left(\mathbf{Z}_{+} ; U\right)$ if and only if $\mathcal{D}_{\phi_{P}} \bar{\pi}_{+} \ell^{2}\left(\mathbf{Z}_{+} ; U\right) \subset \ell^{2}\left(\mathbf{Z}_{+} ; U\right)$ if and only if $\operatorname{dom}\left(\mathcal{D}_{\phi_{P}} \bar{\pi}_{+}\right)=\ell^{2}\left(\mathbf{Z}_{+} ; U\right)$, where

$$
\operatorname{dom}\left(\mathcal{D}_{\phi_{P}} \bar{\pi}_{+}\right):=\left\{\tilde{u} \in \ell^{2}\left(\mathbf{Z}_{+} ; U\right) \mid \mathcal{D}_{\phi_{P}} \bar{\pi}_{+} \tilde{u} \in \ell^{2}\left(\mathbf{Z}_{+} ; U\right)\right\} .
$$

$\mathcal{D}_{\phi_{P}} \bar{\pi}_{+}$is closed (see [9, Lemma 27]). It follows from the Closed Graph Theorem [3, Theorem II.I.9] that $\mathcal{D}_{\phi_{P}} \bar{\pi}_{+}$is bounded because its domain is complete. Conversely, a domain of a closed operator is complete only if the operator is bounded, by [3, Remark II.I.3].

So it remains to prove that the conditions of (68) and (69) are equivalent. By I/Ostability of $\Phi$, the sequence $\left\{\left\langle J\left(C x_{k}+D u_{k}\right),(-,,-)\right\rangle_{Y}\right\}_{k \geq 0}$ in equation (58) is absolutely summable. But then $\left\{\left\langle\Lambda_{P}^{-1}\left(-Q_{P} x_{k}+\Lambda_{P} u_{k}\right),(-,,-)\right\rangle_{U}\right\}_{k \geq 0}$ is absolutely summable if and only if $\left\{\left|P\left(x_{k}, x_{k}\right)-P\left(x_{k+1}, x_{k+1}\right)\right|\right\}_{k \geq 0}$ is absolutely summable, just by looking at equation (58). This completes the proof.

So by Proposition 41, only the condition in claim (iii) of Theorem 40 that $\phi_{P}$ should be outer with bounded inverse remains less concrete. It is easy to see that for power stable systems this follows from the familiar requirement that $P($,$) should be a (power)$ stabilizing solution of the Riccati equation: if both $\rho(A)<1$ and $\rho\left(A+B K_{P}\right)<1$ then $\phi_{P}$ is both I/O-stable and outer (see [12], [13]). For infinite dimensional power stable result we refer to e.g. [4], [14].

Let us briefly reiterate from [17] the classical results for the existence of outer factorizations in the case when the Popov operator $\bar{\pi}_{+} \mathcal{D}^{*} J \mathcal{D} \bar{\pi}_{+}$is positive (and the input space $U$ is separable). It is well known that such Popov operators arise e.g. in the study of linear quadratic optimal control problems and in the factorization versions of Bounded and Positive Real Lemmas (see [23, Section 8]).

By [17, Theorem 3.4], a positive self-adjoint Toeplitz operator $\bar{\pi}_{+} T \bar{\pi}_{+}$has a factorization $\bar{\pi}_{+} T \bar{\pi}_{+}=\bar{\pi}_{+} \mathcal{X}^{*} \mathcal{X} \bar{\pi}_{+}$with outer $\mathcal{X}$ if and only if it has a factorization $\bar{\pi}_{+} T \bar{\pi}_{+}=\bar{\pi}_{+} A^{*} A \bar{\pi}_{+}$ where $A$ is some bounded, causal and shift invariant operator. Such an operator always exists if the Toeplitz operator is coercive: $\bar{\pi}_{+} T \bar{\pi}_{+}>\epsilon \mathcal{I}$ for $\epsilon>0$ by [17, Theorem 3.7]. See also [22, Lemma 11] and [26, Proposition 4.2., p.201 and Remark, p.204 ].

If we have $\bar{\pi}_{+} T \bar{\pi}_{+}=\bar{\pi}_{+} \mathcal{D}^{*} J \mathcal{D} \bar{\pi}_{+}>>0$ (i.e. positive and coercive), then the existence of a bounded outer factor $\mathcal{X}$ follows. A trivial sufficient condition is for the positive cost functional $J>>0$ with $\Phi J$-coercive. 


\section{References}

[1] R. Curtain and J. Oostveen. Riccati equation for strongly stabilizable bounded linear systems. Submitted, 1997.

[2] R. Curtain and H. Zwart. An introduction to infinite-dimensional linear systems theory, volume 21 of Texts in Applied Mathematics. Springer Verlag, New York, Berlin, 1995.

[3] S. Goldberg. Unbounded Linear Operators; Theory and Applications. Dover Publications, Inc., 1985.

[4] A. Halanay and V. Ionescu. Time-varying discrete linear systems, volume 68 of Operator Theory Advances and Applications. Birkhäuser, Basel, Boston, Berlin, 1994.

[5] J. W. Helton. A spectral factorization approach to the distributed stable regulator problem; the algebraic Riccati equation. SIAM Journal of Control and Optimization, 14:639-661, 1976.

[6] V. Ionescu and M. Weiss. Continuous and discrete-time Riccati theory: a Popovfunction approach. Linear Algebra and Applications, 193:173-209, 1993.

[7] P. Lancaster and L. Rodman. Algebraic Riccati equations. Clarendon press, Oxford, 1995.

[8] J. Malinen. Minimax Control of Distributed Discrete Time Systems through Spectral Factorization. Proceedings of EEC97, Brussels, Belgium, 1997.

[9] J. Malinen. Well-posed Discrete Time Linear Systems and Their Feedbacks. Helsinki University of Technology Institute of Mathematics Research Reports, A384, 1997.

[10] K. Mikkola. On the stable $H^{2}$ and $H^{\infty}$ infinite-dimensional regulator problems and their algebraic Riccati equations. Helsinki University of Technology, Institute of mathematics, Research Report A383, 1997.

[11] B. P. Molinari. Equivalence relations for the algebraic Riccati equation. SIAM Journal of Control, 11(2):272-285, 1973.

[12] B. P. Molinari. The stabilizing solution of the discrete algebraic Riccati equation. SIAM Journal of Control, 11:262-271, 1973.

[13] B. P. Molinari. The stabilizing solution of the algebraic Riccati equation. IEEE Transactions on Automatic Control, 20:396-399, 1975.

[14] J. Oostveen and H. Zwart. Solving the infinite dimensional discrete time algebraic Riccati equation using the extended symplectic pencil.

[15] T. Pappas, a. J. Laub, and N. R. Sandell Jr. On the numerical solution of the discrete-time algebraic Riccati equation. IEEE Transactions on Automatic Control, 25(4):631-641, 1980.

[16] H. J. Payne and L. M. Silverman. On the discrete time algebraic Riccati equation. IEEE Transactions on Automatic Control, AC-18(3):226-234, 1973. 
[17] M. Rosenblum and J. Rovnyak. Hardy classes and operator theory. Oxford university press, New York, 1985.

[18] W. Rudin. Functional Analysis. McGraw-Hill Book Company, New York, TMH edition, 1990.

[19] O. J. Staffans. Quadratic optimal control of stable systems through spectral factorization. Mathematics of Control, Signals and Systems, 8:167-197, 1995.

[20] O. J. Staffans. Quadratic optimal control of stable well-posed linear systems. Transactions of American Mathematical Society, 349:3679-3715, 1997.

[21] O. J. Staffans. Coprime factorizations and well-posed linear systems. SIAM Journal on Control and Optimization, 36:1268-1292, 1998.

[22] O. J. Staffans. Feedback representations of critical controls for well-posed linear systems. International Journal of Robust and Nonlinear Control, 8:1189-1217, 1998.

[23] O. J. Staffans. Quadratic optimal control of well-posed linear systems. SIAM Journal of Control and Optimization, 37:131-164., 1998.

[24] O. J. Staffans. On the distributed stable full information $H^{\infty}$ minimax problem. International Journal of Robust and Nonlinear Control, 8:1255-1302, 1999.

[25] A. Stoorvogel. The $H^{\infty}$ control problem: a state space approach. University of Michigan, Department of Electrical Engineering and Computer Science, Ann Arbor, USA, 1995.

[26] B. Sz.-Nagy and C. Foias. Harmonic Analysis of Operators on Hilbert space. NorthHolland Publishing Company, Amsterdam, London, 1970.

[27] G. Weiss. Regular linear systems with feedback. Mathematics of Control, Signals, and Systems, 7:23-57, 1994.

[28] G. Weiss. Transfer functions of regular linear systems, Part I: Characterizations of regularity. Transactions of American Mathematical Society, 342(2):827-854, 1994.

[29] M. Weiss. Riccati equation in Hilbert space: A Popov function approach. $\mathrm{PhD}$. Thesis. The University of Groningen, 1994. 